\title{
ISOCRATE ET LA QUESTION DES MERCENAIRES
}

\author{
Christian Bouchet
}

\begin{abstract}
Résumé
Quand il évoque les mercenaires, Isocrate les traite soit comme des soldats, combattants vaillants, soit comme des stipendiés, contraints par la misère à louer leurs services, soit comme des étrangers (le そ́ćvo peut alors signifier le mercenaire), soit enfin comme des vagabonds. Si les jugements qu'il porte sur ces hommes sont parfois très durs, impitoyables, il faut noter aussi - et c'est ce sur quoi j'insiste ici - que le

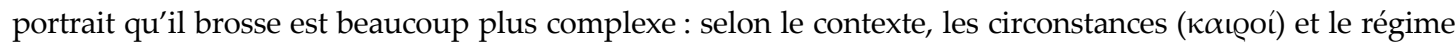
politique impliqué, il se fait accusateur ou bien plus humain, prenant en compte les difficultés économiques et familiales de ces individus.
\end{abstract}

Quand Isocrate envisage la guerre à mener contre les Perses (les barbares), il ne peut éviter la question des troupes à engager : s'agira-t-il de citoyens, censés être dévoués corps et âme à leur cité ? S'agira-t-il, aussi, de ces mercenaires qui écument les campagnes et qui sont prêts à se vendre au plus offrant, de ces mercenaires qui souffrent autant qu'ils font souffrir les Grecs ? Il a fallu attendre nos années 70 pour voir les historiens s'intéresser véritablement au mercenariat ${ }^{1}$. Avant, hormis quelques études comme celle de H. W. Parke², le soldat stipendié a été souvent oublié ou traité rapidement. Il n'est qu'à considérer les ouvrages portant sur Isocrate, tel celui de Georges Mathieu ${ }^{3}$. Le terme de mercenaire ne figure pas une seule fois dans l'étude - il est vrai, inachevée - de Paul Cloché4.

Et, pourtant, la position d'Isocrate face à ce phénomène non seulement militaire, mais aussi économique, social, politique et culturel qu'est le mercenariat, en essor au IVe siècle, mérite une analyse, une approche renouvelée. Je tâcherai donc de montrer ici comment le rhéteur désigne, explicitement ou non, le mercenaire, et surtout quelle est son opinion politique (Isocrate est-il isolé au IVe siècle ?), enfin, ce qui n'a guère été traité jusqu'ici, son sentiment, pour ne pas dire sa sensibilité. Le mercenaire n'est pas (seulement) la brute qui pille et qui tue.

Une relecture attentive de tous les textes $\mathrm{d}^{\prime}$ Isocrate $^{5} \mathrm{~m}^{\prime}$ amène donc à reconsidérer la question. Sans limiter l'analyse aux termes qui peuvent désigner les mercenaires, mais en prenant en compte les allusions et les périphrases, je pense montrer l'évolution de l'image du mercenaire chez Isocrate, et, finalement, sa complexité. La difficulté

\footnotetext{
${ }^{1}$ Parmi les études à signaler : Hunt 2007, Bettalli 1992, 2006a et b, Trundle 2004, Bearzot 2001, Ducrey 2000, Garlan 1999, Yalichev 1997, Burckhardt 1996, Krasilnikoff 1992 et 1993, Pritchett 1992, Marinovič 1988, Aymard 1967.

2 Parke 1933.

${ }^{3}$ Mathieu 1925: dans le chapitre consacré au Sur la Paix, seules quelques lignes mentionnent les mercenaires, pp. 114-115, 120 et 123. Voir aussi p. 163. J'ajouterai que la position d'Isocrate sur la question des mercenaires est très rapidement évoquée chez Yalichev 1997, qui présente ainsi l'orateur : «Isocrates hated mercenaries in general and mercenaries who served the Persians in particular », p. 127, ou encore, dans sa nomenclature : «Athenian orator and bitter critic of Greek mercenary service », p. 284.

${ }^{4}$ Cloché 1963.

${ }^{5}$ Tous les textes cités sont tirés du Thesaurus Linguae Graecae. Les traductions sont miennes.
} 
de cette démarche, d'abord lexicale, est qu'il n'existe pas, en grec classique, un terme qui signifie à tous les coups "mercenaire", pas même le mot misthophoros. Il faudra dès lors toujours se montrer prudent et tâcher de distinguer les troupes militaires dont parle Isocrate : grecques composées de citoyens, grecques formées de mercenaires ou d'alliés, mixtes, non grecques?

Dans un ouvrage récent, Matthew Trundle a répertorié et analysé tous les termes qui, pour un Grec, pouvaient, non pas nommer formellement, mais au moins évoquer les mercenaires ${ }^{6}$. Son étude, précieuse et minutieuse, $\mathrm{s}^{\prime}$ attache cependant plus aux historiens (Hérodote, Thucydide et Xénophon pour les Ve et IVe s.) qu'aux orateurs (exception faite de Démosthène). Isocrate y est peu présent, et pourtant, la question du vocabulaire du mercenaire se pose chez lui. C'est en revanche l'un des grands mérites du livre de Ludmila Marinovič ${ }^{7} \mathrm{~d}^{\prime}$ avoir insisté sur le traitement et le vocabulaire du mercenariat dans les textes d'Isocrate.

Je partirai d'un classement lexical en quatre catégories, qui, pour être sans doute schématique, n'en a pas moins l'avantage de mettre en lumière la manière dont Isocrate a pu appréhender le phénomène : le mercenaire est 1 . un combattant, 2 . un salarié, 3. un étranger, 4 . un vagabond, un déraciné. Ces désignations ne s'excluent d'ailleurs pas forcément les unes les autres. Il faudra compter aussi avec les nombreuses périphrases et allusions aux mercenaires, si bien qu'il serait d'une méthode hasardeuse que de s'en tenir aux vocables utilisés. Les contextes et les intentions rhétoriques sont enfin au moins aussi importants, et il est évident que la vision du mercenaire que nous trouvons dans le Sur la Paix en 356 n'est pas celle que nous lisons dans

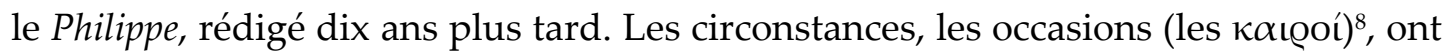
changé ; la définition du mercenaire a évolué.

\section{Le combattant}

D'une manière technique, les mercenaires peuvent être présentés comme des troupes d'abord d'appoint, puis plus nombreuses, voire majoritaires selon les cas, donc comme des soldats. La difficulté est que nous ne savons pas toujours, quand Isocrate parle de soldats, s'il pense à des citoyens ou à des étrangers, ou encore aux deux à la fois. Le terme $\sigma \tau \varrho \alpha \tau \iota \omega \tau \eta \varsigma^{9}$ renvoie en effet aussi bien aux premiers qu'aux seconds. Les $\sigma \tau \varrho \alpha \tau \iota \tilde{\omega} \tau \alpha \iota$ peuvent être des citoyens se battant pour leur propre cité10, ou contre le pouvoir en place, comme ce fut le cas à Samos ${ }^{11}$ en 411 . Ils peuvent être aussi des

\footnotetext{
${ }^{6}$ Trundle 2004, spécialement pp. 10-21 pour la terminologie.

${ }^{7}$ Marinovič 1988, voir chapitre VII, pp. 237-269, « Le mercenariat et la Grèce [Le mercenariat comme problème général de la Grèce] », avec les discours d'Isocrate comme source principale. Voir aussi Burckhardt 1996, pp. 180-196, «Isokrates », même s'il ne s'agit pas de la seule question du mercenariat, l'approche de la question étant essentiellement politique.

${ }^{8}$ Sur l'importance de cette notion de kairos, voir Trédé 1992.

${ }_{9}^{9}$ Voir Trundle 2004, p. 10 : le terme stratiōtēs est le mot neutre qui désigne tout type de soldat en grec classique. Chantraine 1968 signale que le mot stratos (sv) désigne «l'armée installée, qui campe ».

${ }^{10}$ Busiris, 19, et Aréopagitique, 7, à propos des soldats lacédémoniens.

11 Sur l'attelage, 16, 19 et 20.
} 
alliés. Dans le Panégyrique, 185, Isocrate mentionne précisément le recrutement de « soldats "12, dont on peut penser, vu le contexte, qu'il s'agit de citoyens ou d'alliés, bien plutôt que de mercenaires, quoique les deux types de combattants soient souvent associés. Un tel recrutement est extrêmement douloureux lorsque les cités luttent les unes contre les autres, comme durant la guerre de Corinthe; il ne le sera pas quand il s'agira de partir combattre le grand Roi. Dans le Panégyrique, le schéma est clair, quasi dichotomique: d'un côté des troupes grecques, commandées par les Athéniens et les Lacédémoniens, d'un autre côté, les barbares.

Quarante ans plus tard, le Panathénaïque, 79-82 qui emprunte au fonds de l'épopée l'exemple d'Agamemnon, montre bien le flou qui entoure la définition de ces $\sigma \tau \varrho \alpha \tau \iota \tilde{\omega} \tau \alpha$ t: il n'est pas aisé de dire si ces hommes qu'Agamemnon prenait dans

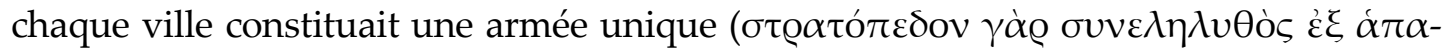
$\sigma \tilde{\omega} \nu \tau \tilde{\omega} \nu \pi \delta ́ \lambda \varepsilon \omega v, 81)$, ou une fédération d'alliés. La tradition, largement développée par notre rhéteur lui-même, indique en tout cas qu'Agamemnon a recruté une armée grecque, une armée dont il a été le général en chef (Panathénaïque, 76-77) ${ }^{13}$. Cependant, l'emploi du terme $\mu \iota \sigma \theta$ oфo@ó, sur lequel je reviendrai, suggère assez clairement qu'Isocrate pense ici aux mercenaires semblables à ceux du IVe siècle:

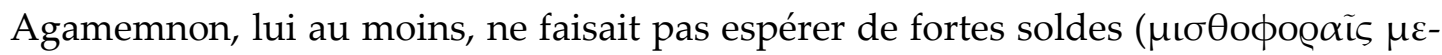
$\gamma \alpha \dot{\lambda} \alpha \iota \varsigma, 82)$ à ses hommes, qu'il entretenait à partir des ressources de l'ennemi.

Un passage semble malgré tout opérer une distinction : dans la Lettre II $A$ Philippe, 9, les soldats sont, sans doute, à distinguer des mercenaires, qui sont, eux,

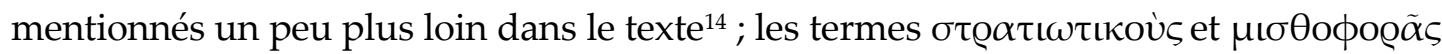
$\varepsilon ँ v \varepsilon \kappa \alpha$ renvoient certainement à deux groupes d'hommes, les premiers constituant les troupes «classiques » ou bien signifiant les soldats de façon générique, les seconds, de façon plus formelle, renvoyant aux mercenaires. Toutefois, hormis ce texte, il arrive que les $\sigma \tau \varrho \alpha \tau \iota \tilde{\omega} \tau \alpha \iota$ soient des mercenaires ${ }^{15}$, des citoyens recrutés par

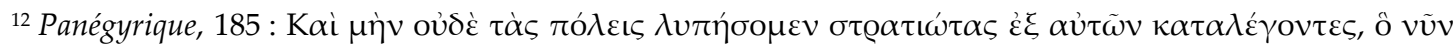

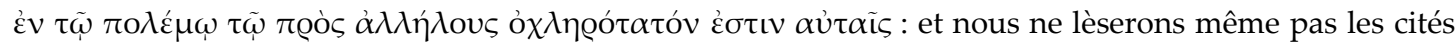
en recrutant chez elles des soldats, ce qui, aujourd'hui, dans la guerre à laquelle elles se livrent entre elles, leur est extrêmement pénible.

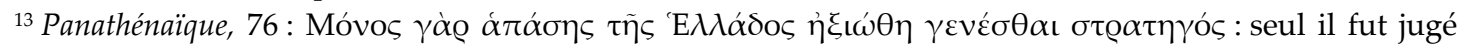
digne de devenir le général de la Grèce tout entière, et $77: \sigma \tau \varrho \alpha \tau o ́ \pi \varepsilon \delta o v ~ \delta \varepsilon \dot{~ \sigma v \sigma \tau \eta ́ \sigma \alpha \varsigma . ~ V o i r ~ a u s s i ~ P a n e ́-~}$

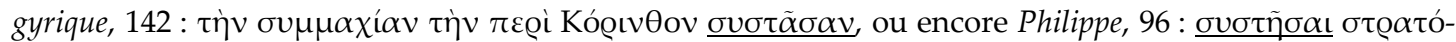

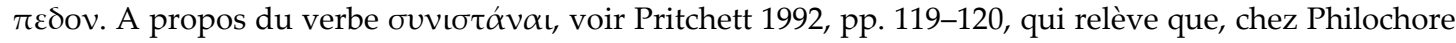

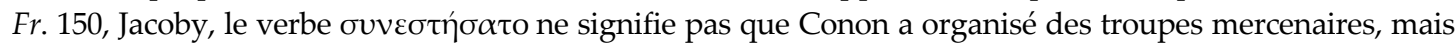

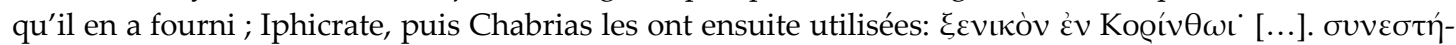

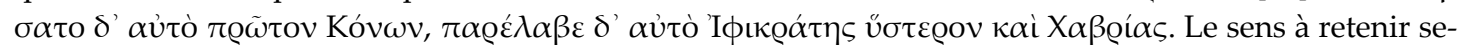
rait bien de réunir, de rassembler des troupes disparates au départ. Pour dire « disposer » ses troupes sur

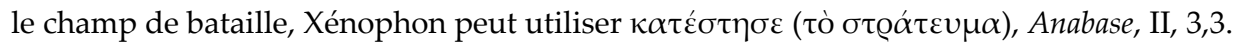

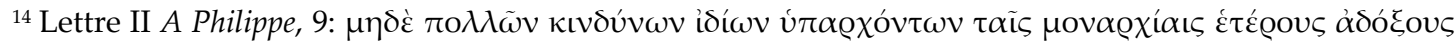

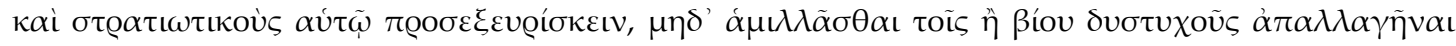

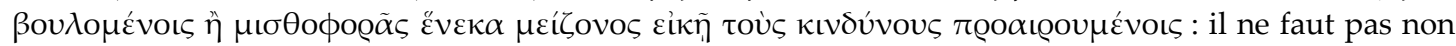
plus, alors que les régimes autoritaires comportent bien des risques, s'en créer d'autres, de ceux, sans gloire, que courent les simples soldats, ni rivaliser avec ceux qui veulent en finir avec une vie de malheurs ou qui, avec l'espoir d'une solde plus importante, choisissent aveuglément les risques.

${ }^{15}$ Je note par ex. l'emploi de stratiōtēs chez Aristote, qui désigne les mercenaires, opposés aux troupes de

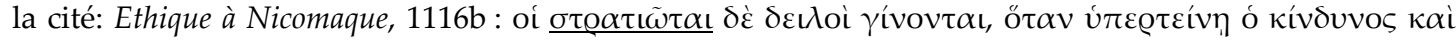

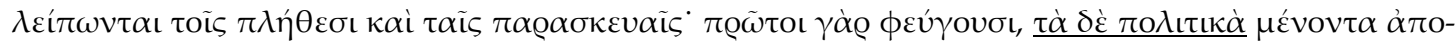
$\theta v \eta ́ \sigma \kappa \varepsilon \iota$ : ces soldats sont lâches, lorsque le danger est trop grand et qu'ils sont inférieurs en nombre et en armement ; ils sont les premiers à fuir, alors que les troupes de la cité meurent sur place. Hunt 2007
} 
tel ou tel chef pour des opérations particulières : ainsi en est-il dans le Panégyrique, où il est question des troupes de Conon en Asie mineure et du versement de leur solde ${ }^{16}$.

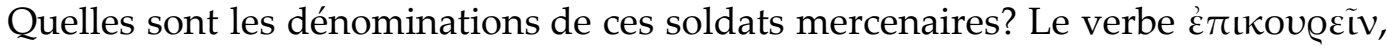
désignant le fait de servir en tant qu'auxiliaire, peut renvoyer aux troupes mercenaires $^{17}$ (comme c'est déjà le cas, avant Isocrate, chez Hérodote ${ }^{18}$ et Thucydide ${ }^{19}$ ). Ce sens est bien attesté dans l'Eginétique, 38 où il est question d'auxiliaires engagés pour attaquer Siphnos. Dans le Panégyrique, 147, le mot éтíkov@oı désigne les mercenaires ${ }^{20}$ engagés contre Artaxerxès II et commandés, selon Isocrate, par huit généraux; dans le $§ 168, \dot{\varepsilon} \pi$ « texte indique que des hommes sont contraints par la pauvreté ( $\delta \iota^{\prime} \varepsilon ̌ v \delta \varepsilon \iota \alpha \nu \tau \tilde{\omega} \nu \kappa \alpha \theta^{\prime}$

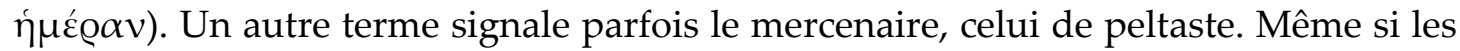
deux mots ne se recoupent pas, le soldat légèrement armé (le peltaste) n'étant pas forcément un mercenaire et ce dernier pouvant combattre comme hoplite, non comme peltaste, Isocrate les confond à l'occasion ${ }^{21}$. Ainsi dans le Panégyrique 115, 141, 144, le Sur la Paix, 118, le Sur l'Echange, 111.

Comment ces forces, ainsi définies de façon plus ou moins claire, sont-elles perçues? La guerre entreprise par Cyrus contre son frère Artaxerxès II a permis aux mercenaires grecs de montrer leur bravoure et leur efficacité au combat, dit Isocrate dans le Panégyrique, à la suite de Xénophon ${ }^{22}$. Isocrate va encore plus loin dans leur célébration

indique, après Parke, qu'à la fin du IVe siècle, «the term stratiōtēs, previously a neutral term for soldier, meant mercenary and it was the citizen-soldier who needed to be indicated with a modifier», p. 14 .

${ }^{16}$ Panégyrique, 142 : le grand Roi n'a pas payé pendant quinze mois les soldats mercenaires : $\pi \varepsilon v \tau \varepsilon \kappa \alpha i ́ \delta \varepsilon \kappa \alpha$

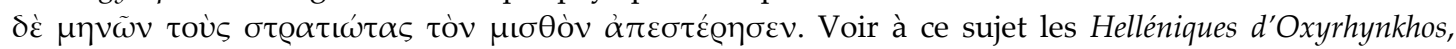
XIV, 2 (Jacoby).

${ }^{17}$ L'épikouria peut aussi désigner simplement l'aide, l'appui militaire, sans qu'il soit question de mercenaires : ainsi dans l'Eloge d'Hélène, 40, Busiris, 22, Archidamos, 63 et 79. Voir Garlan 1999, qui souligne le sens très probable d'auxiliaire du mot épikouros, p. 148, n. 5. Trundle 2004 note p. 13 que, selon Parke 1933, épikouros était un euphémisme pour désigner le mercenaire. Il ajoute, pp. 13-14, que le terme épikouros a tendance à disparaître en cet emploi au IVe siècle : Xénophon ne l'utilise que 2 fois dans l'Anabase, et encore, pour signifier l'aide, l'assistance, et non le mercenaire lui-même. Selon Trundle, seule une occurrence des Helléniques, VII, 1, 23, utilise épikouros pour désigner les mercenaires, d'Arcadie.

${ }_{18}$ Par exemple Hérodote, I, 64, 2, à propos des mercenaires de Pisistrate.

${ }^{19}$ Par exemple Thucydide, I, 115, 4, au sujet des événements de Samos en 440-439.

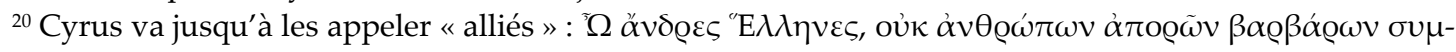

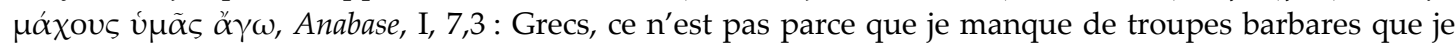
vous prends pour alliés. L'idée exprimée par Cyrus est que les mercenaires grecs sont plus courageux que les barbares.

${ }^{21}$ Le texte de Plutarque, Agésilas, 22, 2, peut être ambigu, quand il décrit la défaite subie par les Lacédémo-

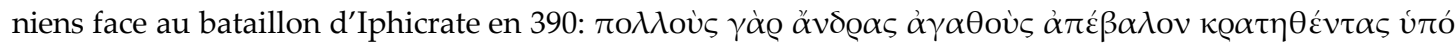

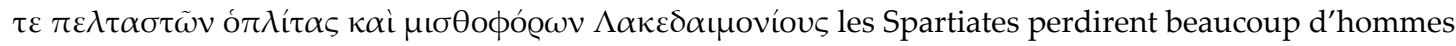
de valeur, avec la défaite d'hoplites face à des peltastes, de Lacédémoniens face à des mercenaires. Les

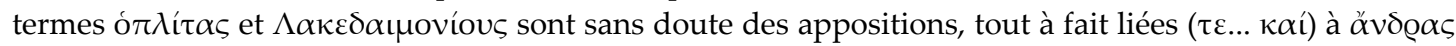
$\dot{\alpha} \gamma \alpha \theta$ oùs. La phrase signifierait alors que des hoplites ont été vaincus par des peltastes, et que des Lacédémoniens (= hoplites) l'ont été par des mercenaires (= peltastes). Voir Xénophon, Helléniques, IV, 5, 12-16 pour le récit de cette bataille. A propos des peltastes chez Xénophon, voir Riedinger 1991, pp. 223-225.

22 Panégyrique, 144 et 145 pour les mercenaires de Cyrus. Pour le nombre de mercenaires grecs à Counaxa, voir Xénophon, Anabase I, 7, 10. Sur la question - encore très discutée aujourd'hui - de l'antériorité du Panégyrique par rapport à l'Anabase, voir Nouhaud 1982, p. 323 : Isocrate peut avoir écrit avant Xénophon et s'être servi d'une autre source, à savoir Ephore. Quant à la question de l'efficacité du mercenaire, voire de sa supériorité militaire par rapport au citoyen, se reporter au discours de Polydamas à Sparte en 374, 
lorsqu'il écrit qu'ils n'étaient pas venus en Asie pour piller ${ }^{23}$. La rhétorique, qui dédouane ces troupes de toute accusation de rapine systématique, est évidemment trop appuyée ici pour que l'on puisse adhérer à cette affirmation, et l'on sait bien comment les mercenaires vivaient du pays qu'ils occupaient ${ }^{24}$. L'intention d'Isocrate est de montrer comment ces troupes ont pu, après avoir mené la guerre contre le grand Roi luimême, se replier sans difficultés. En même temps, le lecteur sera bien obligé de constater que le portrait - sincère ou non, de circonstance ou non - des mercenaires n'est pas toujours à charge, au contraire. Nous en avons là un premier indice. Plus tard, le rhéteur reprendra ce thème des soldats mercenaires, conduits par Timothée ${ }^{25}$ ou encore par Cléarque contre Artaxerxès II ${ }^{26}$.

Le mercenaire est donc d'abord un combattant. Isocrate ne va guère plus loin dans sa définition militaire, et il avoue lui-même son peu de compétence dans ce domaine. Il se déclare en effet étranger à la pratique et à la science de la stratégie ${ }^{27}$. Cela ne l'a toutefois pas empêché d'écrire sur ce sujet : dès le Busiris, Isocrate traite de questions militaires ${ }^{28}$. De plus, il brosse des années plus tard dans le Sur l'échange (101-139) le portrait du stratège idéal, attaché à l'intérêt de sa cité, sous les traits de Timothée ; enfin, il se permet de donner des conseils à Philippe ${ }^{29}$. Cela étant dit, son approche de la question du mercenariat est autre, plus financière et politique que proprement militaire.

citant les propos de Jason de Phères ; le tagos se vante de disposer de troupes aguerries et endurantes (Xénophon, Helléniques, VI, 1, 5).

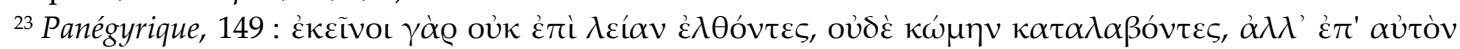

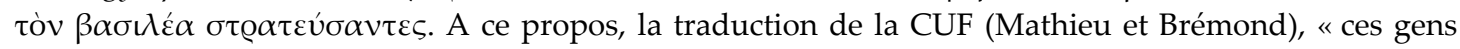
qui n'étaient pas venus (seulement) pour piller et n'avaient pas fait qu'occuper une bourgade... », me semble devoir être corrigée. Il n'y a pas nécessité d'ajouter «seulement» et «ne... que ». Cela fausse d'ailleurs le sens du texte, qui est plutôt : « ces gens qui n'étaient pas venus pour piller et qui ne s'étaient pas même emparés d'un village ».

${ }^{24}$ Dans l'Anabase, Xénophon insiste largement sur la question du ravitaillement des mercenaires. Il convient de distinguer plusieurs modes, selon le pays traversé et selon que ces troupes sont victorieuses ou non. Les mercenaires pouvaient acheter leurs denrées sur des marchés (par ex. I, 5,6), ou bien se livrer au pillage (I, 1,19). En position de vaincus, ils devaient obtenir l'autorisation de piller, seulement s'ils ne trouvaient rien à acheter (II, 3,26, ou II, 4, 27). Pour la question du ravitaillement auprès des marchands ou du pillage, voir Krasilnikoff 1992, p. 24 et 1993, pp. 84-89.

${ }^{25}$ Sur l'échange, 120, 124. Pour Timothée, voir infra.

${ }^{26}$ Philippe, 91, 96 et 125-126 (où Isocrate mentionne ces soldats - stratiōtas - que les Perses font venir de Grèce).

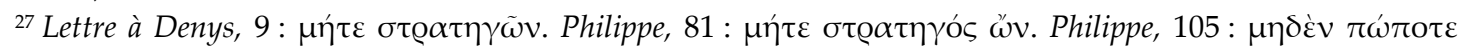

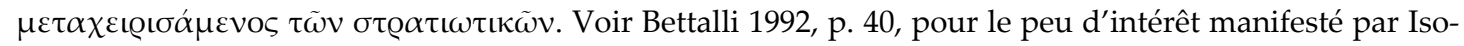
crate à l'égard de la stratégie et de la tactique.

${ }^{28}$ Busiris, 15-18.

${ }^{29}$ A partir du $\S 30$ du Philippe, après de multiples précautions oratoires (voir §§ 18-21). La majeure partie de ce discours consiste en une leçon de géopolitique - selon Isocrate - et en une analyse des exigences de paix et de concorde (en Grèce) et de guerre (contre le Roi). 


\section{Le «salarié»}

Le mot français mercenaire contient en lui-même l'idée d'un prix à payer, d'un salaire. Ce prix, en grec, apparaît dans le terme $\mu$ toӨós (l'indemnité) ${ }^{30}$, auquel il faudra

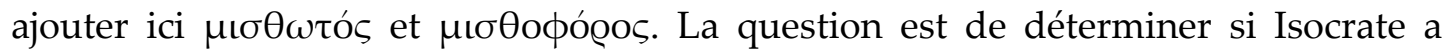
utilisé ce substantif et ses dérivés pour mentionner des troupes mercenaires. Le $\mu \iota \sigma-$ $\theta \omega \tau$ tó s est-il un mercenaire ? Même si un passage de Thucydide ${ }^{31}$ nous permet de considérer que le $\mu \iota \sigma \theta$ ó $\varsigma$, versé aux mercenaires, qu'ils soient grecs ou non, distingue ces hommes et les troupes civiques, il demeure difficile de répondre d'une manière tranchée. Pierre Ducrey a, de son côté, étudié la solde versée aux soldats, qu'ils fussent citoyens ou mercenaires ${ }^{32}$.

Chez Isocrate, la première occurrence du terme $\mu$ toӨós figure, dès 402-401, dans le C. Callimakhos, 60, et renvoie à la solde des matelots, payée exceptionnellement après la défaite d'Aigos-Potamoi, en 405, par le client d'Isocrate et son frère. Rien ne dit formellement que ces matelots aient été (tous) des mercenaires ${ }^{33}$. Le récit de Xénophon mentionne, dans la flotte opposée à Lysandre en 405, des marins athéniens, qui, une fois faits prisonniers, furent condamnés à mort par les alliés de Lysandre et exécutés ${ }^{34}$. Ensuite, le terme $\mu$ IбӨós apparaît dans le Sur l'attelage, 20, et, là encore, nous ne pouvons pas dire qu'il évoque le mercenariat: Alcibiade, en 411, désireux de

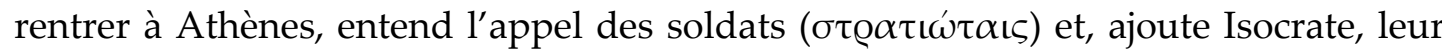
distribue une solde sur ses propres fonds. Il ne peut s'agir que des soldats athéniens basés à Samos. Dans le Panégyrique, 142 (avec un rappel au $\S 153$ ), il est question d'un $\mu$ tбӨós, que le grand Roi, Artaxerxès II, n'aurait pas versé durant quinze mois (entre 396 et 394) aux soldats ( $\sigma \tau \varrho \alpha \tau \iota \omega ́ \tau \alpha \varsigma)$ commandés par Conon ${ }^{35}$. Quelles sont ces troupes ${ }^{36}$ ? Le texte d'Isocrate précise que, si Conon n'était pas intervenu, ces hommes se seraient dispersés $(\delta\llcorner\varepsilon \lambda v ́ \theta \eta \sigma \alpha v)$. Ce verbe a toutes les chances de signifier

\footnotetext{
30 Voir Will 1975, qui souligne le sens archaïque de misthos : la récompense, plutôt que le salaire. Il note aussi, p. 432, la connotation péjorative que peut revêtir le mot dans la bouche des oligarques de 411. Le misthos, qui désigne l'indemnité, est peut-être déjà assimilé à l'argent que l'on verse à des mercenaires. Pour l'emploi de ce mot au IVe s. voir Mossé 1976 : le misthos «désigne dans l'Athènes du IVème siècle la rétribution de toutes les fonctions publiques et même de la présence aux séances de l'ecclésia », pp. 9798. A propos de la terminologie de cette indemnité ou de ce paiement, voir Krasilnikoff 1993, pp. 77-79 pour les différents termes (misthos, sitos, sitēresion, trophē, eis sitēsin), principalement chez Xénophon, mais aussi chez Isée, Démosthène et [Aristote], pp. 91-95.

31 Thucydide I, 60, 1-2 : en 432, les Corinthiens envoient à Potidée des contingents, sans doute réguliers

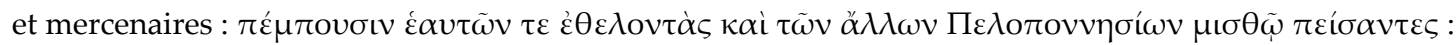
les Corinthiens envoient des volontaires de chez eux ainsi que d'autres Péloponnésiens, qu'ils avaient séduits par une solde.

${ }^{32}$ Ducrey 2000, voir en particulier le tableau des soldes versées, p. 199. Voir aussi Will 1975, qui renvoie à Athenian Tribute Lists II, 34 par ex. pour signaler que le misthos était le terme « officiel pour désigner la rémunération des soldats athéniens à l'époque de la guerre du Péloponnèse », p. 433, note 3. A propos des rapports pouvant exister entre le pillage et le salaire, voir Krasilnikoff 1992: 32-33: le butin provenant du pillage pouvait être vendu par les chefs de façon à assurer le versement du misthos. Ce fut le cas par ex. avec Agésilas et Dercylidas.

${ }^{33}$ Pour Marinovič 1998, p. 239, note 7, «il est possible [...] qu'on ait également en vue les mercenaires (composant probablement une partie des marins) ».

${ }^{34}$ Xénophon, Hell., II, 1, 32.

35 Voir supra.

36 Voir Pritchett 1992, pp. 118-121.
} 
que les troupes étaient composites, en partie régulières, en partie mercenaires ${ }^{37}$. La difficulté est qu'Isocrate se refuse à présenter Conon comme un chef de mercenaires.

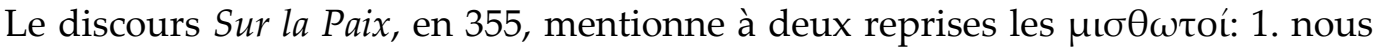

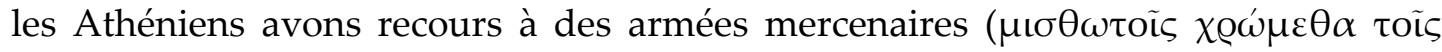

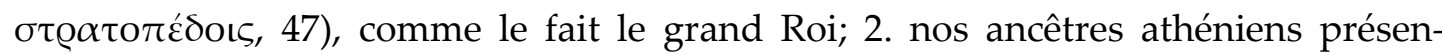
taient à leurs alliés au théâtre le spectacle des sommes qu'ils avaient prélevées sur

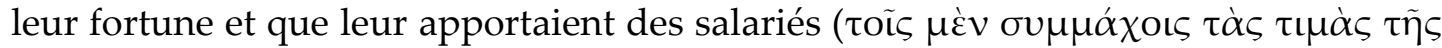

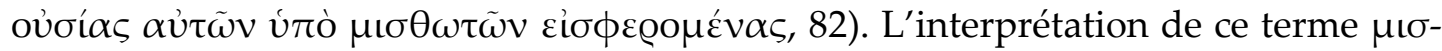
$\theta \omega \tau$ oí est difficile : dans le premier passage, du fait même qu'il se rapporte à $\sigma \tau \varrho \alpha-$ $\tau 0 \pi \varepsilon ́ \delta o ı \varsigma ~ l ' a d j e c t i f$ désigne très certainement des mercenaires, dont l'image est associée à des pratiques barbares, celles du grand Roi. Dans le second, il est beaucoup plus ambigu et rien n'autorise à le traduire par «mercenaires » : le substantif risque bien de signifier "des hommes payés pour cette tâche », des "salariés ", de la cité s'entend, même si Isocrate peut jouer sur les mots et laisser penser à son lecteur que des «mercenaires » sont à l'origine de cet argent ${ }^{38}$. Nous n'avons donc qu'un emploi assuré chez Isocrate de $\mu$ I $\theta \omega \tau$ oí au sens de «mercenaires », et encore ce sens est-il

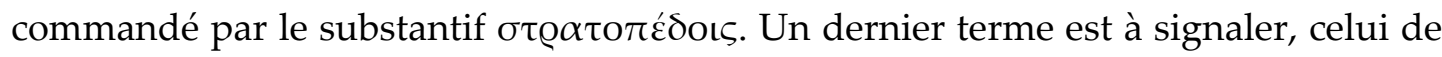
$\mu \iota \sigma \theta$ oфóoo $\varsigma^{39}$, au $\S 112$. L'adjectif qui qualifie ici des «individus» signifie sans aucun doute mercenaires, - et non pas seulement des troupes « recevant une solde »-, dans la mesure où ces hommes sont censés être des étrangers, inconnus jusque-là des monarques qui les emploient ${ }^{40}$. Il est utile de souligner que, dans ce cas, Isocrate envisage des $\mu \iota \sigma \theta$ oфó@o intervenant dans un régime de type tyrannique, comme il visait au $\S 82$ le fonctionnement du pouvoir perse. Le recours à des mercenaires, outre le coût qu'il induit ${ }^{41}$, risque bien d'être présenté par Isocrate comme une déviance de la démocratie, qui s'aligne sur le modèle de régimes autoritaires dans lesquels les citoyens ne se battent pas ou plus pour eux-mêmes.

Dans le Sur l'échange, Isocrate consacre un long développement en forme d'éloge à Timothée (§§ 101-139) ${ }^{42}$. Celui-ci a pu soumettre Samos en 366-5 en finançant ses troupes (8000 peltastes et 30 trières) à partir du pays ennemi ${ }^{43}$. Il est manifeste qu'Isocrate

\footnotetext{
${ }^{37}$ Platon, dans le Ménexène, 245a, parle de ces bannis et de ces volontaires qui combattirent à Cnide sous les ordres de Conon. Voir Bouchet 2007, pp. 237 et 239.

38 Papillon 2004, p. 153, n. 47 : «Isocrates seems to imply that the money being carried into the theater by workers was also the money that was brought to Athens through mercenary actions such as are described in section 79 ».

${ }^{39} \mathrm{C}^{\prime}$ est d'ailleurs ce terme, plus méprisant que les autres, qui semble s'imposer au IVe et surtout après pour désigner les mercenaires (Trundle 2004, p. 16, qui cite le Xénophon des Helléniques, Ephore, Diodore et Arrien).

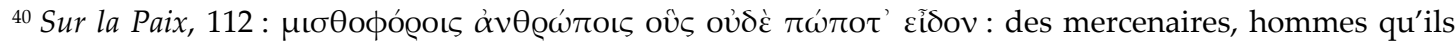
n'ont même jamais vus.

${ }^{41}$ On peut d'ailleurs se demander s'il était vraiment plus coûteux pour une cité d'employer des mercenaires que de payer des troupes de citoyens. Voir Hunt 2007, p. 142.

42 Voir Bianco 2007: « La digressione di Isocrate su Timoteo », pp. 61-89, et « Altre fonti principali su Timoteo », pp. 91-143; Bettalli 1992, pp. 41-44.

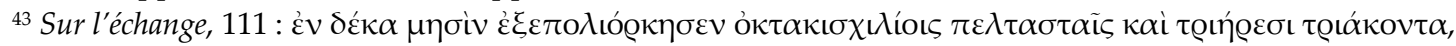

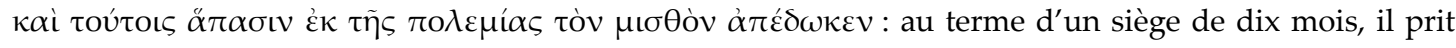
[Samos] avec huit mille peltastes et trente trières, et c'est sur le butin de guerre qu'il leur versa à tous leur solde. La même idée figure dans le § 120 : comme il ne pouvait compter sur le financement de la cité, $c^{\prime}$ est la guerre elle-même qui devait lui permettre de verser une solde pleine et entière à ses troupes : $\delta \dot{\alpha}$
} 
célèbre le fils de Conon pour mieux condamner Charès, dont le portrait est tout à l'opposé de celui de Timothée ${ }^{44}$. L'auteur de l'Argument du Sur la Paix met en évidence le rôle de Charès dans le déclenchement et le déroulement de la guerre des Alliés ${ }^{45}$. Il

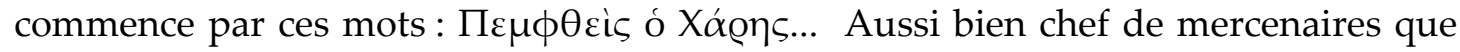
stratège athénien envoyé en Asie, Charès aidait en fait le satrape de Phrygie maritime, Artabaze, et les troupes révoltées contre le grand Roi. D'où l'ultimatum lancé en 355 par ce dernier aux Athéniens, exigeant d'eux le rappel du stratège ${ }^{46}$. Enfin, toujours dans le Sur la Paix, alors qu'il traite des questions militaires, Isocrate recourt à des arguments, non pas techniques (la pertinence de la stratégie et de la tactique adoptée sur le terrain par exemple), mais moraux, voire intellectuels ${ }^{47}$. Cela signifie sans doute que la question de fond pour Isocrate est la dérive morale de la fonction de stratège, qui est de plus en plus assimilée à celle d'un chef de mercenaires, certes compétent Isocrate ne saurait se prononcer sur ce chapitre -, mais pas ou peu attaché à sa cité. En ce sens, il est probable qu'il vise ici des hommes comme Charès ${ }^{48}$, muni des pleins pouvoirs dans la campagne qu'il mena en Chersonèse ${ }^{49}$ en 357. Isocrate oublie simplement de signaler qu'en se mettant au service de satrapes comme Artabaze Charès a pu payer ses troupes, ce que ne faisait pas la cité athénienne, et que le butin résultant du pillage de Lampsaque et de Sigée a profité à Athènes ${ }^{50}$. De plus, Charès restait placé sous le contrôle de la cité : comment aurait-elle pu le rappeler sans cela ? Quelle est, en face, la situation de Timothée en 366 ? Il vient de rentrer à Athènes, qu'il avait quittée après son procès ${ }^{51}$ de 373 et après s'être mis au service du grand Roi comme commandant de troupes mercenaires en Egypte, ce que tait évidemment Isocrate, qui passe de l'expédition conduite par Timothée autour du Péloponnèse (§§ 109-110) en $375 \mathrm{au}$

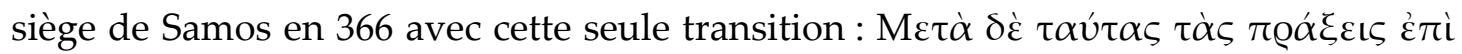

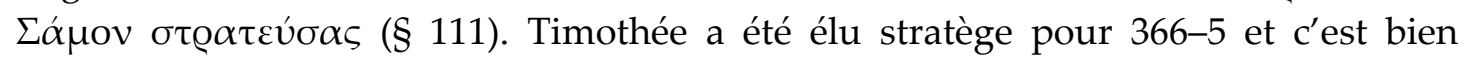
missionné par les Athéniens qu'il a embarqué, d'abord pour soutenir Ariobarzane, en

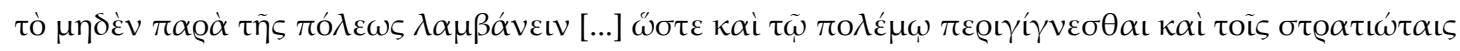

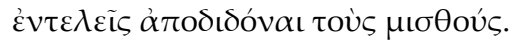

${ }^{44}$ Voir Harding 1995, pp. 105-125: Isocrate aurait rédigé le discours Sur la Paix pour défendre Timothée contre les attaques de Charès. Voir l'analyse de ces attaques portées contre Charès dans Bianco 2003 : Charès est le contre-exemple de Timothée, le premier affecté de tous les vices, le second pourvu de toutes les qualités. Isocrate serait même à l'origine de l'historiographie à charge contre Charès : « La parole di Isocrate contro Carete (...) hanno tuttavia pesato come macigni per tutti i secoli a venire », p. 139.

${ }^{45}$ Cawkwell 1981 souligne, p. 52, la version tendancieuse de cet Argument et analyse au contraire le sentiment de Démosthène.

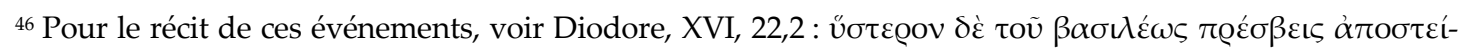

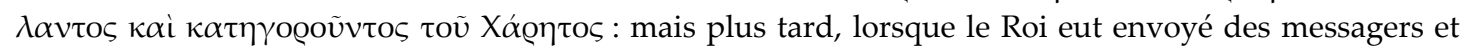
mis en accusation Charès.

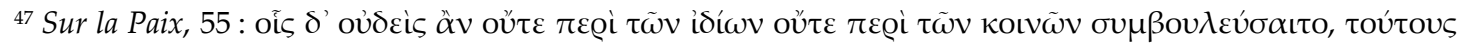

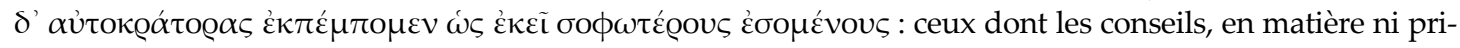
vée, ni publique, n'inspireraient personne, nous les envoyons en tant que stratèges avec les pleins pouvoirs, comme s'ils devaient, une fois là-bas, être plus avisés.

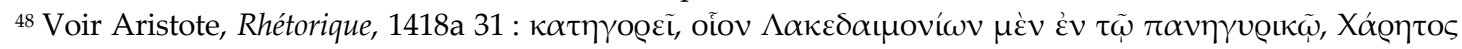

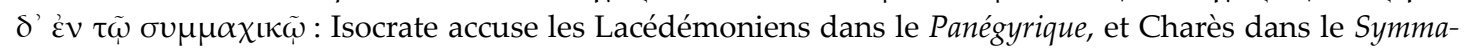
chique (= le Sur la Paix).

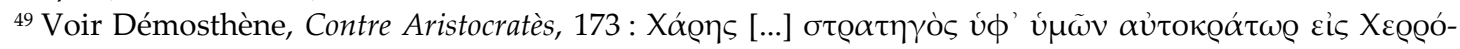
vๆ sonèse.

${ }^{50}$ Voir à ce sujet Pritchett 1991, p. 381 et p. 423, n. 619.

${ }^{51}$ Voir Bianco 2007, pp. 30-35. 
définitive pour s'emparer de Samos. Les troupes qu'il dirige alors peuvent être formées de citoyens et de mercenaires. Isocrate ne précise pas et Timothée ne fait nullement figure de condottière ici, même s'il l'a été et s'il a cherché à constituer ses équipages à partir de mercenaires ${ }^{52}$. En fait, il est souvent difficile au IVe s. de distinguer l'activité privée d'un stratège chef de mercenaires et son activité commandée par la cité, surtout quand ce sont des auteurs comme Isocrate, partisan de Conon et de Timothée, qui traitent de ces questions.

Dans le Philippe, Isocrate mentionne les mercenaires des Thébains, tués en Phocide et pour qui, ajoute-t-il, la mort vaut mieux que la vie (§55). Le terme utilisé est $\mu$ $\sigma \theta 0-$ фóoos, qui s'oppose aux Phocidiens qui ne craignent pas de mourir pour leur patrie $(\text { ibid. })^{53}$. Ce jugement émis par Isocrate a suscité, de la part de L. Marinovič ${ }^{54}$, un commentaire peut-être discutable: la remarque d'Isocrate (ces mercenaires 'pour qui la mort vaut mieux que la vie' est sans doute «faite en passant » et constitue «l'une de ces notations purement rhétoriques qui abondent dans l'œuvre du moraliste qu'était Isocrate ». Je voudrais montrer pour ma part que la commisération n'est peut-être pas absente du texte et que le regard porté par Isocrate n'est pas forcément accusateur. Nous avons là au contraire une forme de pitié et d'indignation, qui, si elle peut relever du topos, n'est pas à négliger.

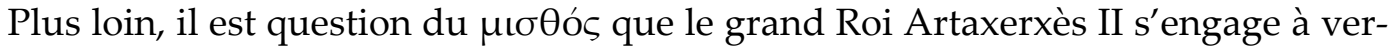
ser intégralement aux soldats qui l'avaient combattu sous les ordres de Cyrus et de Cléarque ${ }^{55}$. Là encore, ce sont des troupes mercenaires qui sont évoquées. Le mot $\mu \mathrm{\sigma} \sigma$ Oós figure enfin au $\S 116$ du Panathénaïque pour désigner le «salaire » ou l' "indemnité » que la cité versait aux plus démunis qui avaient pris l'habitude de trouver en terre étrangère de quoi subsister. S'agit-il de mercenaires ? C'est probable, quoique non dit formellement.

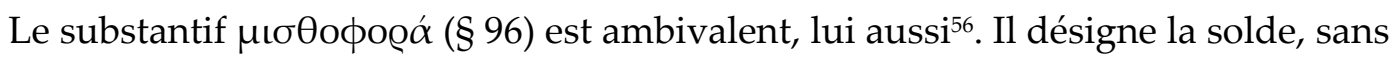
doute des soldats « réguliers ", et non pas des mercenaires. L'idée est que les recruteurs, qui devaient aller chercher des mercenaires dans d'autres cités, coûtaient plus

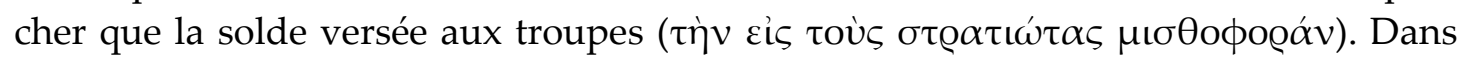
la Lettre II $A$ Philippe, ce même mot de $\mu$ toӨoфo@ó renvoie à coup sûr aux mercenaires, à ces hommes " qui choisissent d'affronter aveuglément les dangers avec l'espoir

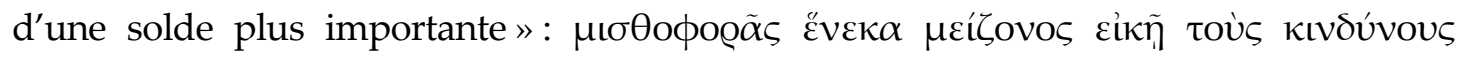

\footnotetext{
52 Xénophon rapporte une différence de méthode entre Timothée et Iphicrate dans le recrutement des équipages (Helléniques, VI, 2, 10-14) : alors qu'il venait d'être élu stratège en 373, Timothée ne parvint pas à réunir les équipages nécessaires à l'expédition prévue autour du Péloponnèse. Les Athéniens le destituèrent et le remplacèrent par Iphicrate, qui, contrairement à Timothée qui avait cherché en vain du côté des îles, fit pression à Athènes même. Sur Iphicrate et Timothée, voir Parke 1933, pp. 50-57 pour Iphicrate, et Pritchett 1992, pp. 119-125.

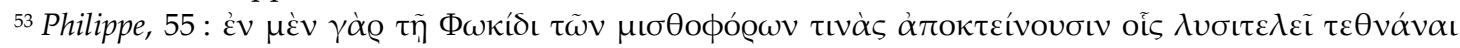

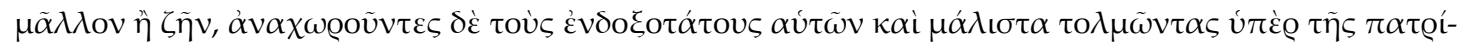

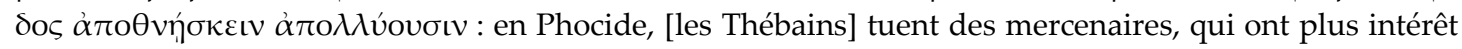
à être morts que vivants, ils perdent les plus glorieux d'entre eux, ceux qui ont tout à fait le courage de mourir pour la patrie.

${ }^{54}$ Marinovič 1988, p. 265.

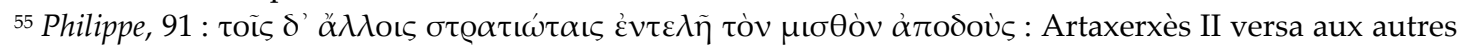
soldats leur salaire intégral.

${ }^{56}$ L'ambiguïté est levée quand le terme misthophoros est accompagné de xénos, comme chez Xénophon, Hel-

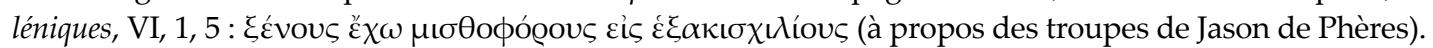




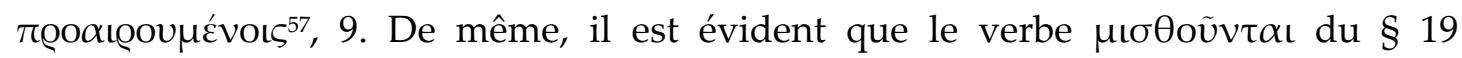
désigne l'action de payer des mercenaires. Enfin, nous avons vu, plus haut, dans le

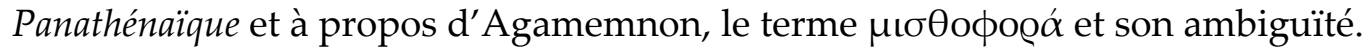

Il ressort donc de cette liste que le mot $\mu$ ıөós et ses dérivés peuvent ou non désigner le salaire des mercenaires. On ne peut en tout cas prendre le risque de le rap-

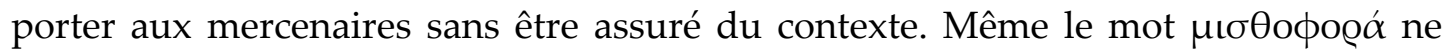
renvoie pas toujours ni nécessairement à la solde des mercenaires : ainsi Démosthène en Olynthienne II, 34 assume-t-il ce terme - qui peut étonner, il est vrai pour une solde militaire à créer pour les citoyens qui, eux-mêmes, participeront à la défense de la patrie ${ }^{58}$.

\section{L'étranger}

Combattant, stipendié, le mercenaire est aussi, et peut-être davantage encore, l'étranger, encore qu'il faille distinguer l'étranger à la cité et l'étranger à la communauté grecque ${ }^{59}$. Avant d'envisager plus longuement le terme $\xi \dot{c} v o \varsigma$, je signalerai l'emploi

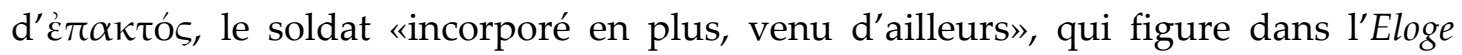
$d^{\prime} H^{\prime} l e ̀ n e^{60}$. $\Xi \varepsilon ́ v o \varsigma$ désigne l'étranger, barbare ou non, soldat ou non. Son sens premier n'est aucunement «mercenaire ». De fait, Isocrate l'emploie à plusieurs reprises, dès 390-385, dans le Busiris 7 et 31, avec l'acception d'étrangers. Peu après, dans le Panégyrique 168, il est clair que l'adjectif ne renvoie pas à l'idée de mercenaire, mais simplement à celle de terre étrangère : les guerres que nous avons provoquées, dit Isocrate,

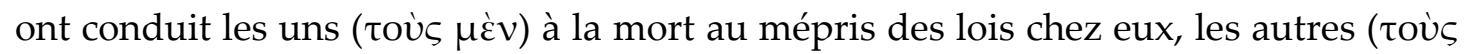
$\left.\delta^{\prime}\right)$ à l'errance à l'étranger avec femmes ${ }^{61}$ et enfants, beaucoup enfin, - troisième caté-

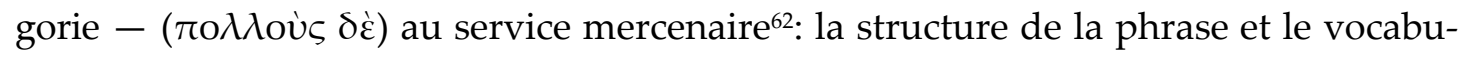
laire peuvent nous inciter à distinguer celui qui erre loin de sa patrie et celui qui, contraint par la pauvreté, doit vendre ses services comme mercenaire. En d'autres termes, les vagabonds et les mercenaires constituent bien, selon la syntaxe, deux groupes différents, à moins de considérer - ce que je ne crois pas - que le $\pi \mathrm{o} \lambda \lambda$ où $\varsigma \delta \dot{\varepsilon} \delta \iota '$

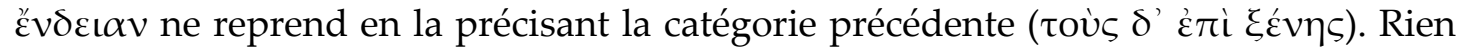
ne nous autorise formellement à le faire. Dans tous les cas, les notions de terre étrangère et de mercenariat restent bien distinctes.

\footnotetext{
${ }^{57}$ Il s'agit là sans doute d'un lieu commun, d'un cliché défavorable. En fait, voir Garlan 1999, p. 172 : les mercenaires ne sont pas tous des aventuriers guidés par l'appât du gain.

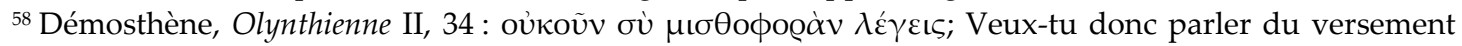
d'une 'solde' ?

${ }^{59}$ Etranger à plusieurs titres, comme l'avait noté Aymard 1967 : les mercenaires sont non seulement des étrangers à la cité ou au roi qui les emploie, mais ils servent dans des armées qui, «camaraderie et esprit de corps à part », leur sont étrangères, p. 487.

${ }^{60}$ Eloge d'Hélène, 37 : Thésée refuse de protéger son pouvoir avec des troupes venues de l'extérieur ; il trou-

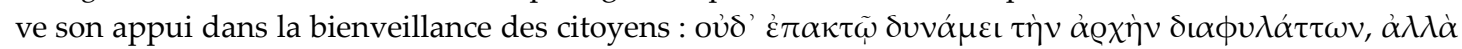

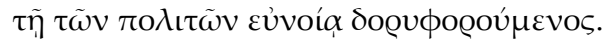

${ }^{61}$ Voir Xénophon, Helléniques, I, 3,19, pour les femmes et les enfants qui accompagnent les mercenaires

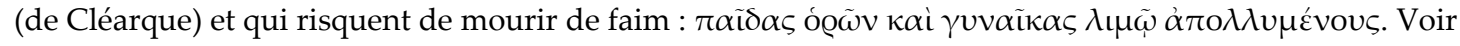
infra pour la question des familles des mercenaires.

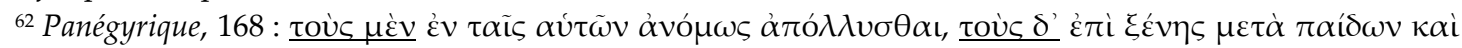

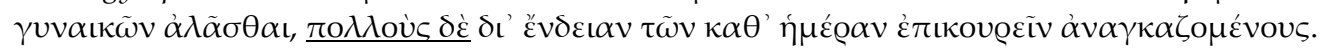


Au contraire, et pour la première fois, nous trouvons dans l'Archidamos 76, l'adjec-

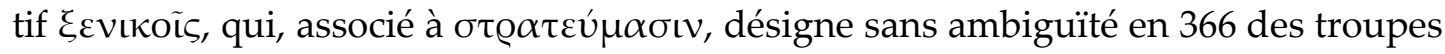
stipendiées ${ }^{63}: 1^{\prime}$ armée dont les Spartiates disposeront en terre étrangère se comportera comme des mercenaires, car exclusivement occupée à la guerre. Cette dernière idée apparaissait d'ailleurs dès le Busiris, 18 : les guerriers de Sparte n'ont d'autre activité que la guerre et sa préparation, les armes et les expéditions ${ }^{64}$, cela à l'imitation des Egyptiens.

Dans le discours Sur la Paix, en 355, le terme ל̌́vos et ses dérivés réunissent clairement les deux sens d'étranger et de mercenaire, comme l'a déjà noté Cynzia Bearzot ${ }^{65}$, qui précise de plus que le pluriel, utilisé dans ce texte, dénonce une masse d'étrangers-mercenaires éminemment dangereux pour la cité66, - alors que le singulier renvoie à l'étranger perçu comme un individu plutôt bien accueilli. Il me semble établi, d'après le contexte, que les '̌ćvou du Sur la Paix désignent les mercenaires, non pas toujours, mais le plus souvent. En effet, les emplois de $\xi \dot{\varepsilon} v o \varsigma$ peuvent ne pas renvoyer à des mercenaires dans deux passages : au $\S 21$, Isocrate évoque les $\xi \dot{v} v o$,

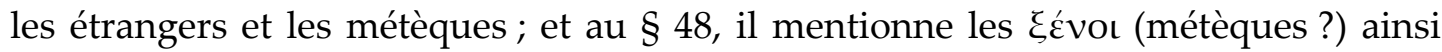
que les esclaves embarqués sur les trières athéniennes. Les trois autres cas sont tous directement reliés à l'idée de mercenaires. Ces trois occurrences explicites ${ }^{67}$ (§§ 24, 46

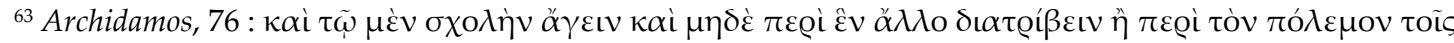

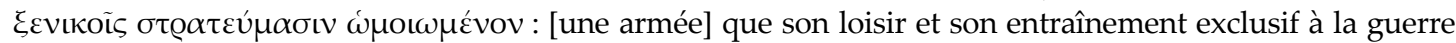
assimileraient à des troupes mercenaires. Ce passage a inspiré un certain nombre de commentaires. Voir par ex. Mossé 1953, qui signale, pp. 31-33, que ces troupes spartiates ne sont pas malgré tout des mercenaires: "Archidamos dit bien qu'il ne s'agit pas d'une semblable armée: ses hommes ne seront pas des mercenaires venus de tous les coins du monde, mais des Spartiates animés par l'ardent amour de la patrie », pp. 31-32. «Il semble qu'on puisse, sans crainte de se tromper, attribuer au seul Isocrate la paternité d'une telle idée. Qu'elle lui ait été inspirée par la conception que se faisaient les Athéniens du régime militaire spartiate, cela ne fait pas de doute», pp. 32-33. Voir aussi Azoulay 2006, qui traite du «modèle ambivalent du mercenariat » : «Isocrate imagine (...) la création d'une armée qui hérite à la fois de la souplesse des corps mercenaires et du patriotisme des armées civiques, sans en avoir aucun des inconvénients : cette communauté utopique serait différente d'une armée de mercenaires en ce qu'elle serait composée de Spartiates animés par un amour ardent de la patrie ; toutefois, elle se distinguerait aussi des troupes civiques ordinaires par son indépendance vis-à-vis de tout « régime organisé » », p. 517.

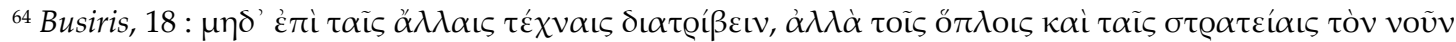

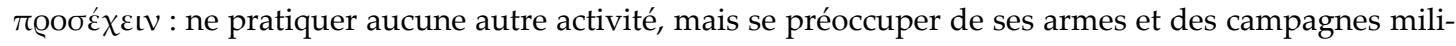
taires.

${ }^{65}$ Bearzot 2001, pp. 53 et 56, analyse l'évolution du vocabulaire chez Isocrate : en 380, « la terminologia è

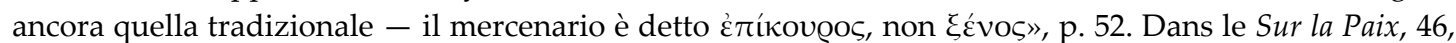
l'Aréopagitique, 9 et Sur l'échange, 116, «la sovrapposizione, nel termine ל́ćvos, dei due significati di « straniero » e « mercenario » in questi passi già evidente », p. 53.

${ }^{66}$ Lengauer 1979, pp. 101-108, qui compte 10 cas de staseis dus à des mercenaires sur les 17 répertoriés (repris dans Ducrey 2000, p. 207). Bearzot 2001, pp. 53-54: «Se lo छ́v́vos come individuo isolato non sembra costituire un problema, giacché si inserisce facilmente nel quadro tradizionale dei rapporti sociali, $\mathrm{i}$

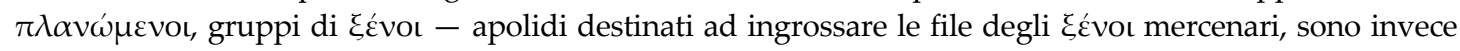
percepti come un fenomeno gravemente destabilizzante ", voir aussi p. 56. Pour Bearzot, si le mercenariat est devenu un phénomène de masse, Isocrate en attribue la responsabilité à Sparte (Panégyrique, 114-

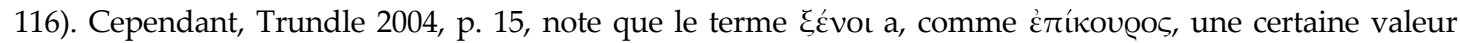
euphémistique. Voir aussi Burckhardt 1996, p. 79, note 13, à propos du

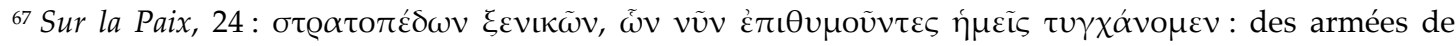
mercenaires que nous nous trouvons désirer aujourd'hui; $46: \xi \varepsilon v o \tau \varrho o \phi \varepsilon i v v ; ~ e t ~ 48: \xi \varepsilon ́ v o เ \varsigma$ ó $\pi \lambda i ́ \tau \alpha ı \varsigma$ $\chi \varrho \omega ́ \mu \varepsilon \theta \alpha$ : nous avons des étrangers pour hoplites.
} 
et 48 bis), à laquelle s'ajoutent des passages plus implicites ( $\$ 79$ par exemple) ${ }^{68}$ sont même délibérément polémiques ${ }^{69}$ et doivent être comprises comme appartenant à un discours qui, en 356/5, vise à convaincre les Athéniens des méfaits de la guerre et

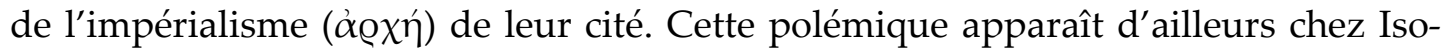
crate dès le Busiris. Il y est écrit en effet qu'en suivant l'exemple des Egyptiens, les

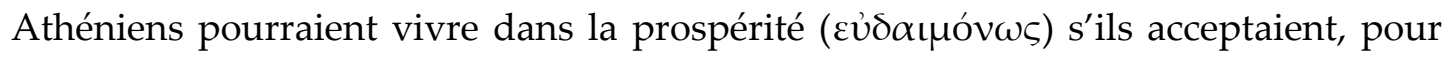
les uns, de travailler, pour les autres, de veiller eux-mêmes aux biens des premiers, donc - implicitement - sans avoir recours à des troupes mercenaires ${ }^{70}$. Même s'il ne faut pas prendre au sérieux ${ }^{71} \mathrm{ni}$ au pied de la lettre l'exercice rhétorique qu'est le Busiris, nous pouvons, avec Christian Froidefond, considérer certaines affirmations comme fondées ou, en tout cas, polémiques, en particulier celles qui touchent aux affaires militaires ${ }^{72}$. Dans le Sur la Paix, le ton est particulièrement violent : les merce-

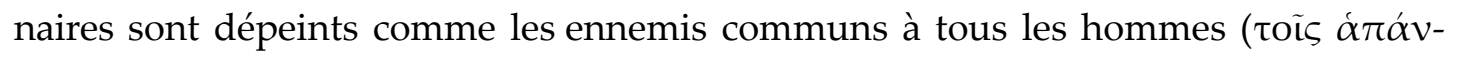

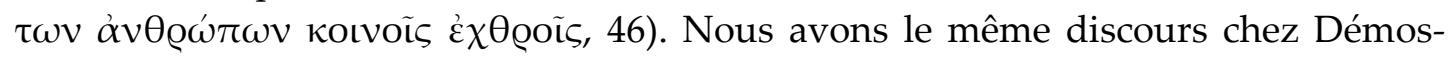
thène ${ }^{73}$ à peine quelques années après, en 352 , ce qui risque d'apparenter la formule à un topos ou à une citation ${ }^{74}$ destiné à marquer les esprits dans telle ou telle circonstance. Il faut surtout noter que le jugement porté par ces deux orateurs ne correspond sans doute pas à l'évolution de l'image du mercenaire au cours du IVe siècle, comme le soulignent Yvon Garlan ${ }^{75}$ et, plus récemment, Marco Bettalli ${ }^{76}$. Il y aurait

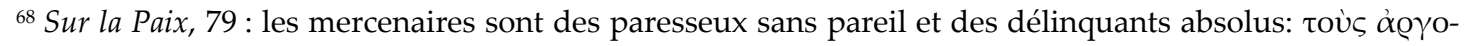

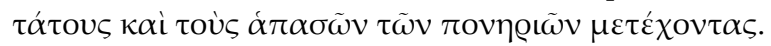

${ }^{69}$ Fuks 1972, p. 29, n. 46 : Isocrate se montre particulièrement dur à l'égard des mercenaires, ici (Sur la Paix, 43-48) plus qu'ailleurs : "Only here does he speak of them with something like hatred »; pour Fuks, ce sentiment serait dû soit à l'emploi (à Athènes) toujours plus fréquent de mercenaires au cours $\mathrm{du}$ IVe siècle, soit à la guerre sociale qu'Athènes mène avec des troupes mercenaires, soit enfin à leurs abus et crimes commis durant cette même guerre.

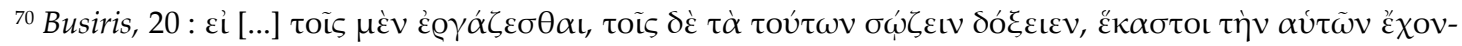

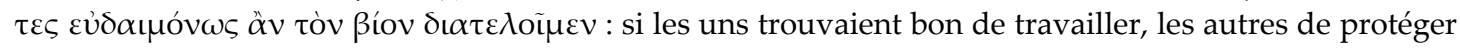
les biens de ceux-là, chacun de nous garderait son patrimoine et mènerait une vie prospère.

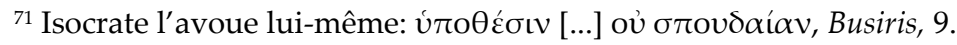

${ }^{72}$ Froidefond 1971 fait remarquer qu'il y a dans cet ouvrage des pages plus sérieuses : ainsi lorsque Isocrate célèbre le fonctionnement militaire de l'Egypte, il dénonce du même coup celui de Sparte, cité trop guerrière (\$§ 19-20). La description de l'Egypte ne jouerait, selon Ch. Froidefond, qu'un « rôle d'utilité ", l'intention d'Isocrate étant de «morigéner Athènes », pour son recours à des mercenaires, « sans exalter Lacédémone », p. 249.

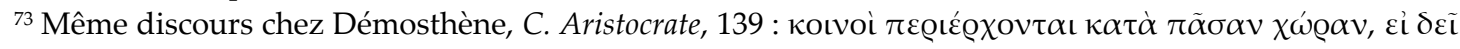

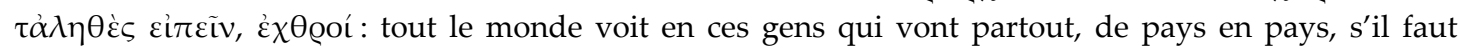
parler vrai, des ennemis.

${ }^{74}$ Ces deux jugements, d'Isocrate et de Démosthène, sont très certainement inspirés d'un vers de Tyrtée,

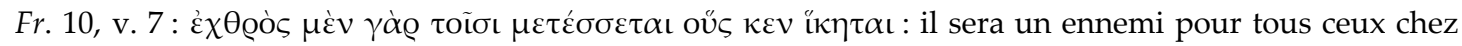
qui il ira.

${ }^{75}$ Garlan 1999, p. 154 : «En règle générale, le mercenaire était d'ailleurs moins perçu comme un étranger que comme un militaire de carrière ». Y. Garlan estime aussi que le mercenaire grec du IVe s préfigure « le type nouveau de quasi-citoyen, de citoyen-sujet qui constituera l'élite politique des royaumes orientaux de l'époque hellénistisque », p. 172.

${ }^{76}$ Bettalli 2006a montre comment des chefs de mercenaires ont pu jouir d'une grande renommée à Athènes, en prenant l'exemple de Léosthène, célébré par Hypéride, p. 22 ; ou encore comment on pouvait ne pas s'indigner, au contraire, d'avoir un parent mercenaire, p. 23 : Eschine écrit dans le Sur l'Ambassade

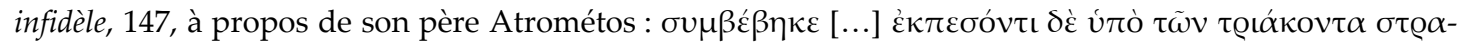

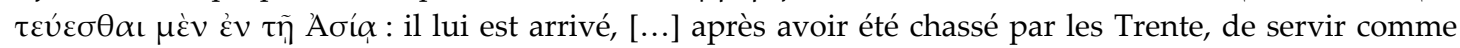
soldat en Asie. Bettalli démontre donc que, contrairement à l'image négative du mercenaire dans les sources
} 
bien un décalage entre Isocrate et la pensée commune du IVe : Isocrate s'arrête de moins en moins sur le côté militaire du mercenaire, alors qu'il insiste sur les aspects ethniques et financiers ou encore économiques (voir mon tableau à la fin de cette étude).

La première occurrence $(\S 24)$ met en avant l'attitude des Athéniens, peu enclins, selon Isocrate du moins ou quelques autres comme Xénophon ${ }^{77}$, à servir eux-mêmes et qui désirent donc employer des mercenaires. Sans chercher à savoir si le recours toujours plus nombreux à des mercenaires n'est pas en vérité lié à l'évolution même de la guerre au IVe siècle, Isocrate préfère alléguer une décadence sociale, voire une démission politique, de ces citoyens qui ne combattent plus eux-mêmes; ce sont eux qui rament, alors que des étrangers servent comme hoplites. Nous avons là l'expression d'un mépris pour les rameurs ${ }^{78}$ (explicité au $\S 48$ ) et pour la flotte, dans un discours, le Sur la Paix, qui dénonce précisément les abus et les dérives de l'hégémonie maritime d'Athènes ${ }^{79}$. De plus, ces pratiques, cette démission du citoyen, ne sont pas celles d'une cité qui peut légitimement aspirer à l'hégémonie, mais plutôt celles des

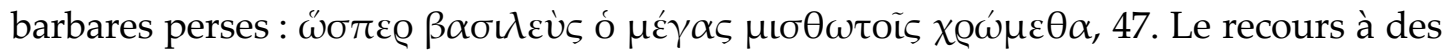
troupes mercenaires signifierait que les citoyens sont lâches ${ }^{80}$ et donc bien inférieurs

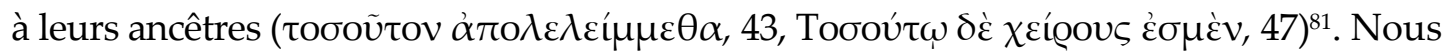
avons là un procédé rhétorique, opposant un autrefois grandiose à un présent décadent, donc un discours hyperbolique qu'il ne faudrait pas prendre au pied de la lettre.

Le deuxième passage $(\S 46)$ implique la question financière et économique, puisqu'il s'agit de nourrir et d'entretenir ces troupes ( $\xi \varepsilon v o \tau \rho o \phi \varepsilon i v)$. Le recours à des mercenaires a un coût, difficilement supportable pour la cité. Cette dépense est d'autant plus

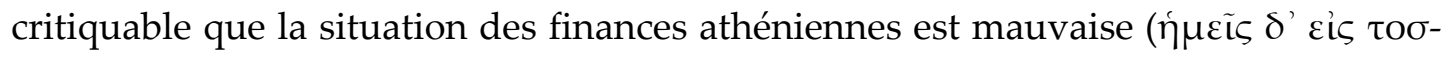
$\alpha u ́ \tau \eta v \dot{\alpha} \pi$ o@í $\alpha v \dot{\varepsilon} \lambda \eta \lambda v \theta$ ó $\varepsilon \varsigma, 47)$. C'est cette contrainte financière, dénoncée par tout un groupe de politiques ${ }^{82}$ dans les années 350 , qui pousserait Athènes à maltraiter

(tourné contre sa cité, une « sorta di metastasi della società », p. 19), ce combattant n'est pas étranger au monde de la cité.

77 Xénophon, qui a personnellement fait l'expérience du mercenariat, écrit à la fin de da vie, dans le Sur

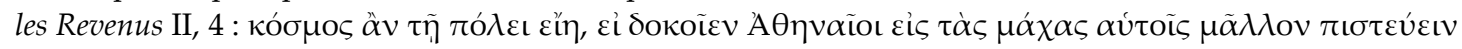

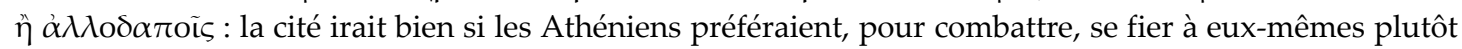
qu'à des étrangers. Dans le chapitre II, 3, Xénophon regrette la promiscuité des barbares et des citoyens

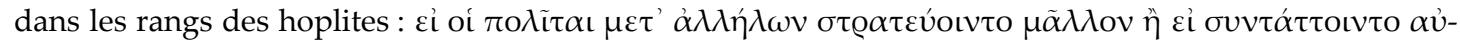

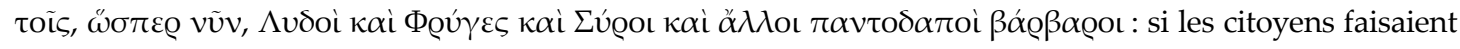
campagne entre eux plutôt que de voir dans leurs rangs, comme aujourd'hui, des Lydiens, des Phrygiens, des Syriens, des barbares de toutes origines.

${ }^{78}$ Sur l'attachement d'Isocrate à l'égard des hoplites et son mépris des rameurs, voir Chankowski 1997, p. 345.

${ }^{79}$ Voir Ceccarelli 1993, qui considère au contraire, pp. 451-455 que, dans le Sur la Paix, Isocrate dénonce l'empire lui-même plutôt que l'empire maritime tout particulièrement.

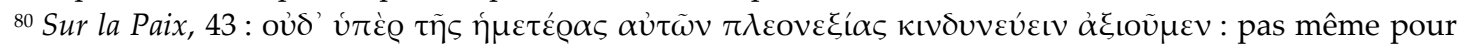

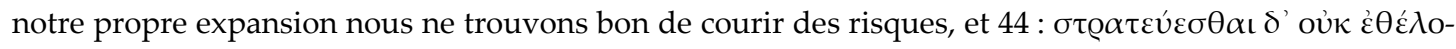
$\mu \varepsilon v$ : nous ne voulons pas partir en campagne. Cela relève d'une vision propre aux orateurs et d'une propagande. En fait, les citoyens restent attachés à la défense de leur cité, voir Chankowski 1997, p. 342.

${ }^{81}$ Déjà, dans l'Eloge d'Hélène, Isocrate indiquait, pour brosser l'anti-portrait de ses contemporains, que Thésée avait avec lui la cité entière pour aller au combat et qu'il refusait des gardes étrangers pour assurer

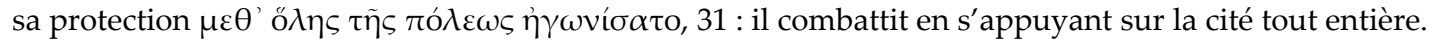

${ }^{82}$ Romilly 1954, p. 342, montre que les différents groupes, modérés ou non, dont nous trouvons écho des programmes politiques chez Démosthène, Xénophon et Isocrate, étaient d'accord pour dénoncer le recours aux mercenaires et réclamer une armée de citoyens, mais qu'ils se distinguaient fondamentalement les 
aussi bien ses alliés ${ }^{83}$ que les citoyens les plus riches ${ }^{84}$ : le recours aux mercenaires, sans être lui-même à l'origine de la guerre des Alliés, a pu détériorer les relations entre Athènes et des cités davantage sollicitées sur le plan financier, soit officiellement soit par des chefs de mercenaires, obligés de trouver eux-mêmes de quoi payer leurs troupes.

La troisième et dernière occurrence $(\S 48)$, reprenant la première $(\S 24)$ en la renforçant et la précisant, joue sur le rapprochement de termes antinomiques dans la société athénienne: ce sont des étrangers qui nous servent d'hoplites. Il faut noter

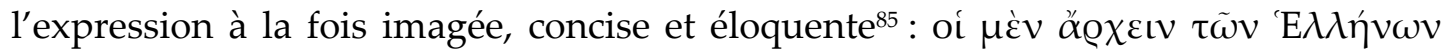

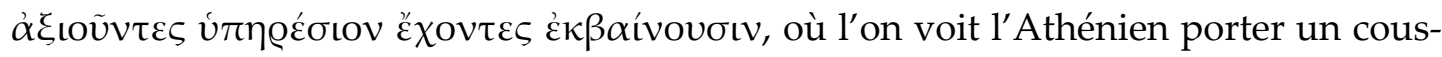
sin de rameur, alors que le mercenaire combat en hoplite, $\mu \varepsilon \theta^{\prime}$ ö $\pi \lambda \omega \nu$.

Au total, le Sur la Paix manifeste une prise de position très négative d'Isocrate à l'égard des '̌́vol, position qui apparaît également dans l'Aréopagitique, 9, et qui s'appuie sur des considérations financières. Ces troupes coûtent cher à la cité, et sans résultat (Isocrate pense sans doute aux campagnes menées par Charès) ${ }^{86}$. L'attaque se poursuit contre ces troupes étrangères/mercenaires dans le Sur l'échange, 116, avec, cette fois, un point de vue moral : on élit des stratèges, que l'on place souvent à la tête de troupes mercenaires ( $\xi \varepsilon v \iota \kappa o i \check{\varsigma})$, comme si on pouvait attendre de cela quelque chose de convenable ( $\tau \iota \tau \tilde{\omega} v \delta \varepsilon$ óv $\tau \omega \nu)^{87}$. Un tel jugement contredirait formellement l'éloge des mercenaires de Cyrus qu'Isocrate formule dans le Panégyrique, 149, si c'était le même vocabulaire ${ }^{88}$. En fait, notre rhéteur ne place pas son lecteur sur le même terrain : nous avions d'un côté, en 380, la célébration de troupes qui ont combattu contre le grand Roi ( $\sigma \tau \varrho \alpha \tau \varepsilon v ́ \sigma \alpha \nu \tau \varepsilon \varsigma)$, d'un autre côté, vers 354, la condamnation d'étrangers

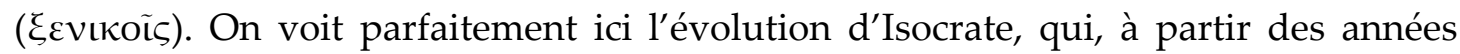
350 , fait plus de cas de l'origine ethnique des mercenaires (voir mon tableau à la fin de cette étude).

Le point de vue est très différent dans le Philippe. Ni la situation ni le destinataire ne sont les mêmes en 346: Athènes n'a plus à lutter contre ses alliés en révolte et le discours s'adresse, non plus aux Athéniens, mais au roi de Macédoine. Le mercenariat concerne donc non plus une démocratie, mais une royauté. Autrefois, dit Isocrate, il

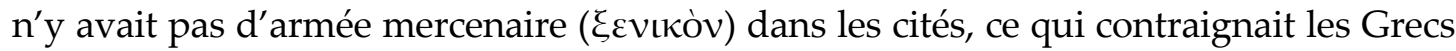
à dépenser une fortune pour aller recruter, parfois très loin, ce genre de troupes

uns des autres par leurs objectifs : «ce qui, chez les orateurs patriotes, est une réforme liée à l'idée de lutte nationale, se présente, chez les modérés, comme un des éléments d'un système d'économies ».

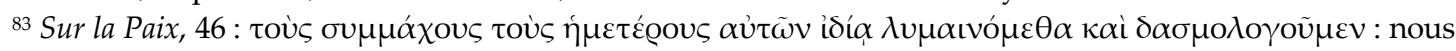
recourons à la violence pour tourmenter nos propres alliés et les exploiter. J'adopte la leçon de l'édition de Mandilaras 2003, en lisant, non pas ióí $\alpha$, mais $\beta$ í $\alpha$, qui, selon moi, donne plus de force au texte en privilégiant l'idée de violence, là où F. Blass 1885 et G. Mathieu 1928, chacun avec une lecture différente, insistaient sur les victimes de ces violences (nous tourmentons chacun de nos alliés).

${ }^{84}$ Isocrate se serait fait le porte-parole de ces citoyens les plus riches : voir Cloché 1963, pour qui il a été « un défenseur résolu, souvent même ardent et véhément, des classes possédantes », p. 99.

${ }^{85}$ Sur la Paix, 48 : ces hommes qui prétendent commander aux Grecs débarquent avec leur coussin de rameurs.

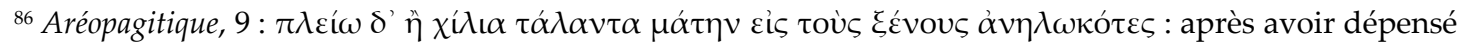
plus de dix mille talents, en vain, pour les mercenaires.

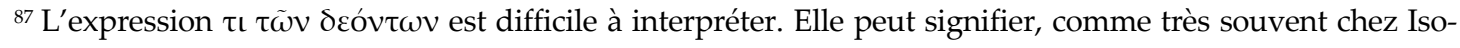
crate, « ce qui convient », ou bien « ce que l'on est en droit d'attendre de troupes militaires », c'est-à-dire des victoires servant l'intérêt de la cité.

88 Voir supra, note 23. 


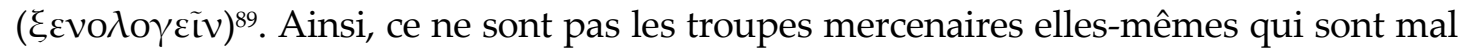
jugées dans le Philippe, mais ce serait plutôt leur absence - affirmation étonnante de la part d'un auteur qui condamne l'association étroite entre la cité démocratique et la pratique du mercenariat. Dans ce même discours (§ 122), les étrangers/mercenaires

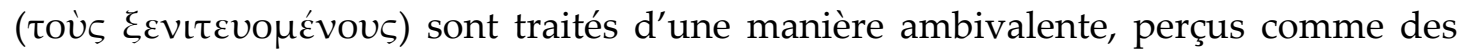
bourreaux ${ }^{90}$ certes, que Philippe devrait tourner contre les barbares (perses), mais aussi comme des victimes. Isocrate cherche à convaincre Philippe d'éloigner des cités grecques ces étrangers, mais nous ne pouvons pas ignorer que les mercenaires sont peut-être même les premiers à souffrir de la pauvreté, qui les oblige à louer leurs services. A moins de penser que nous ayons là un procédé rhétorique destiné à apitoyer Philippe sur le sort de ces mercenaires - ce que je ne peux croire -, ce passage est tout à fait clair, et il nous interdit de considérer qu'Isocrate ne fait que condamner et accuser ces hommes. Cela dit, dans la Lettre II A Philippe, légèrement postérieure au Philippe, la charge contre les mercenaires et ceux qui les emploient est tout à fait explicite $^{91}$. Outre le coût qu'ils représentent, ces mercenaires se révèlent plus souvent nuisibles qu'utiles ${ }^{92}$. Il est évident, si nous confrontons ces deux derniers passages, qui ne se contredisent d'ailleurs pas (on peut souffrir soi-même et se révéler nuisible), que le regard posé par Isocrate sur la question du mercenariat n'est pas figé. Au contraire, son approche est plus complexe qu'il n'y paraît.

Enfin, dans le Panathénaïque - espèce de dialogue entre le maître et un disciple laconophile - , Isocrate dénonce le comportement de certains combattants (les Spartiates), qu'il compare à des troupes mercenaires et qu'il assimile à des hommes mauvais. En effet, le contexte fait que l'expression, on ne peut plus vague, $\mu \varepsilon \gamma \alpha \dot{\lambda} \alpha \alpha$ เ $\delta v \alpha \alpha-$ $\mu \varepsilon \iota \varsigma$, renvoie à des armées semblables à des troupes mercenaires, opposées aux troupes civiques selon le critère suivant: celui du choix ou non de risquer sa vie pour la patrie $^{93}$. Seuls les soldats «honnêtes » ou « dévoués » le font; les autres (ici les Spartiates) se comportent comme des mercenaires en étant prêts à mourir pour s'accaparer le bien d'autrui ${ }^{94}$.

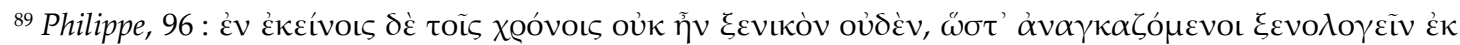

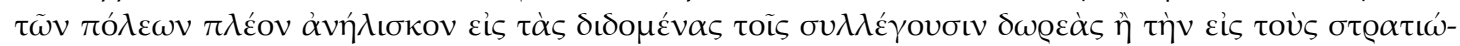

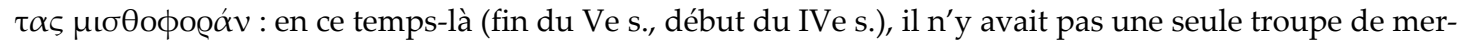
cenaires, si bien que, contraints d'aller en chercher dans les cités, ils dépensaient plus pour récompenser les recruteurs que pour verser leur solde aux troupes.

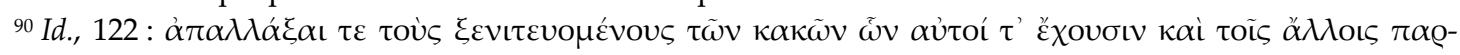

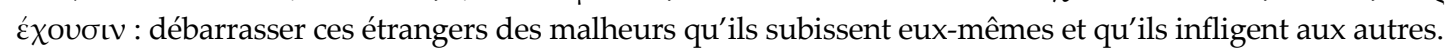

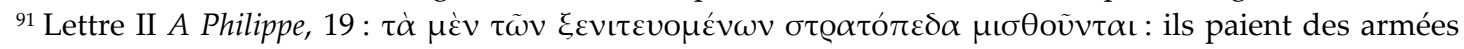
de mercenaires.

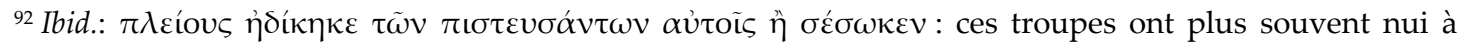
ceux qui se sont fiés à elles qu'elles ne les ont sauvés. Voir Garlan 1999, p. 161 : «Exagération rhétorique, voire obsession pathologique ? Sans doute en partie, mais sur un fond de réalité que nous pouvons illustrer de nombreux exemples concrets ». Voir également, à propos de la capacité de nuisance de ces troupes, l'analyse de Simonetti Agostinetti 2002, pp. 205-216 : pour l'auteur, Alexandre, craignant des mercenaires désoeuvrés à l'issue de la conquête du royaume perse et à leur retour en Grèce, les a installés en Asie mineure.

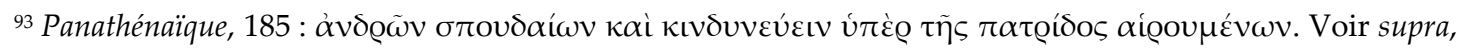
Aristote, Ethique à Nicomaque, 1116b.

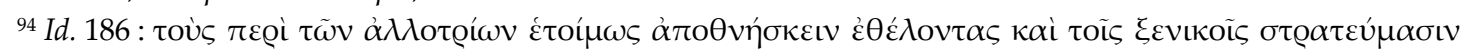

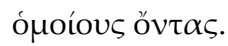


Nous pouvons ainsi penser que le terme $\zeta \bar{c} v o \varsigma$ et ses dérivés signalent l'étranger, et, plus sensiblement à partir des années 350, le mercenaire. Il convient de distinguer alors l'emploi, revendiqué par Isocrate, des mercenaires dans une royauté (Macédoine) et celui, souvent jugé détestable, dans une démocratie (Athènes). L'idée que ce mercenaire est un «déraciné », un étranger à la cité pour laquelle il se bat se retrouve dans l'utilisation du champ lexical de l'errance.

\section{Le déraciné, le vagabond}

Le lexique le plus fréquemment sollicité pour désigner les mercenaires est celui de l'errance ou du vagabondage ${ }^{95}$, que celui-ci inquiète ${ }^{96}$ ou, plus rarement mais de façon certaine, apitoie. Le mercenaire se trouve isolé, comme définitivement éloigné de sa patrie, tel Cléarque, «banni » de Sparte ${ }^{97}$. Il est même remarquable qu'Isocrate n'envisage pas un seul instant le rôle qu'ont pu jouer certains mercenaires (les chefs) dans la vie diplomatique et les relations entre cités ${ }^{98}$. Ces hommes auraient coupé tous les ponts avec les cités, hormis avec celles qui les emploient - et encore. A côté du terme

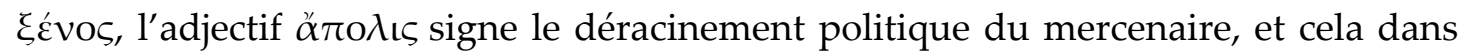

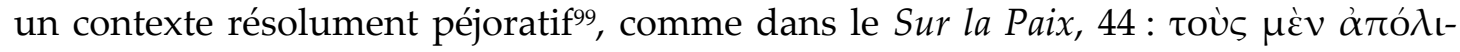

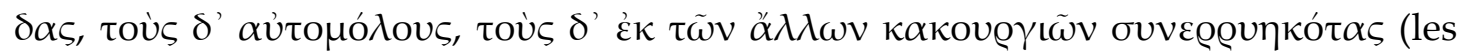
apatrides, les déserteurs et les individus que d'autres crimes ont réunis). J'observerai cependant que $\alpha \ddot{\pi} \pi \mathrm{\prime} \lambda \mathrm{s}$ n'est pas toujours à connotation négative au IVe $\mathrm{s}$. : ainsi chez Xénophon, Sur les Revenus, qui utilise ce terme pour désigner les métèques, ces étrangers qu'il faut attirer en nombre dans la cité100. L'étude des emplois, relativement rares, de cet adjectif à l'époque classique révèle qu'il est essentiellement utilisé par les

\footnotetext{
95 Trundle 2004, p. 34, signale la comédie d'Antiphane du milieu du IVe siècle, Le Soldat, 200-203, chez Athénée, 6, 258. Le mercenaire est un vagabond à la poursuite de la fortune. Voir Démosthène, C. Aristocrate, 138-1399, à propos de Charidème, un modèle de chef de mercenaires apatride : X $\alpha \varrho ı \delta \eta \mu \omega \tau \tilde{\omega} \pi o ́ \lambda ı v$

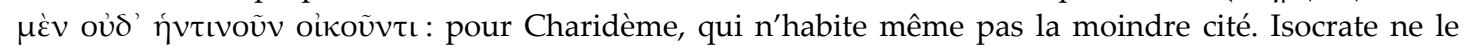
nomme pas, mais il n'est pas exclu qu'il ait pensé à lui, qui réunissait tous les travers aux yeux de l'orateur: eubéen, Charidème a joué un grand rôle auprès de Kersoblèpte, puis lors de l'intervention athénienne - vaine - devant Olynthe en 348. Il s'est opposé à la Macédoine, avant de partir en exil chez les Perses en 338. Pour une présentation de Charidème, voir Parke 1933, pp. 125-132.

${ }_{96}^{6}$ Voir Bettalli 2006b, pp. 57 et 62 (décrire les mercenaires comme des « terroristi » est une exagération, une « forzatura »).

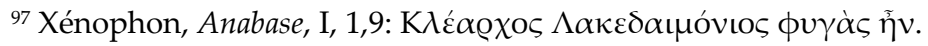

${ }_{98}$ Pour les rapports entre mercenaires et patrie, voir les études de Trundle 2004, chapitre « Networks and Relationships », spécialement p. 150 : «Mercenary service, if indeed it can be called such, served the Greek poleis as an important branch of international diplomacy and policy ».

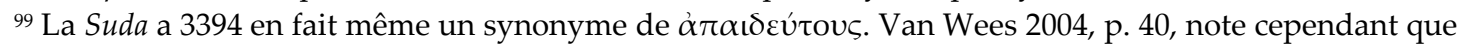
la différence entre mercenaires, immigrants et pirates n'est pas claire pour tout le monde au IVe s. Pour l'image très négative de ces mercenaires, voir, plus tard, à propos des troupes du Spartiate Nabis (fin du

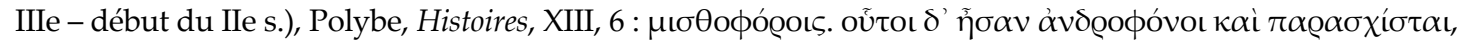

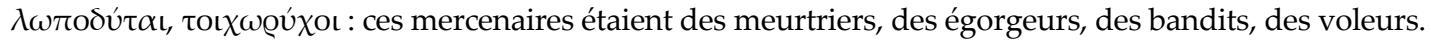

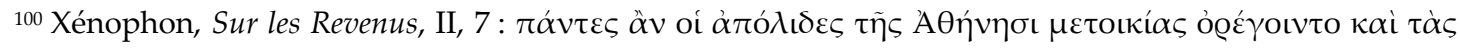

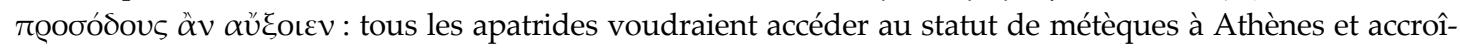
traient ses revenus.
} 
Tragiques, Euripide en tête ${ }^{101}$. Il y a sûrement ici, chez Isocrate, la volonté de marquer les esprits en insistant sur le côté dramatique, voire tragique, de la situation.

Les termes $\pi \lambda \alpha \dot{\alpha} v \eta \varsigma, \pi \lambda \alpha v \omega \dot{\omega} \mu \varepsilon v \varsigma^{102}$, situent les mercenaires en position de rupture, au moins provisoire, avec leur cité d'origine. Ces individus représenteraient l'antithèse même des soldats-citoyens, de ces hommes qui, lors des guerres médiques, se sont battus contre les Perses pour le salut de leur cité et à qui Isocrate rend un hommage appuyé (Panégyrique, 86-98). Les mercenaires, eux, peuvent, à l'occasion, se battre pour le grand Roi contre Evagoras ami et allié d'Athènes (id., 134-135) ${ }^{103}$. Ce même Evagoras, qui dut fuir Salamine de Chypre lors de la prise du pouvoir par le Phénicien Abdémon en 415, jugea avec mépris, à en croire Isocrate, "ces vagabonds, ces

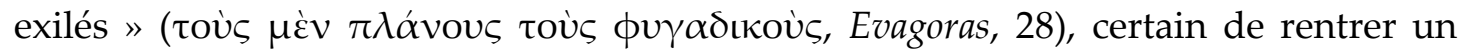
jour à Salamine et d'y exercer le pouvoir absolu. Dans l'Archidamos, si au $\S 17$ il s'agit

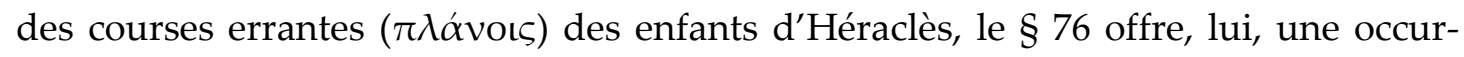
rence du verbe $\pi \lambda \alpha v \tilde{\alpha} \sigma \theta \alpha$ เ bien plus intéressante ici, puisqu'il signifie la capacité de l'armée souhaitée par Archidamos de se déplacer rapidement à travers le territoire ennemi, sans autre base que le terrain propice à ses opérations militaires ${ }^{104}$. En 356, dans la Lettre IX A Archidamos, 9, Isocrate considère que c'est de la folie que de laisser se rassembler des armées plus grandes et plus puissantes à partir de vagabonds qu'à partir de citoyens ${ }^{105}$. Dans le Sur la Paix, Isocrate traite plus largement de la question socio-économique de ces Grecs ${ }^{106}$ (qui ne sont d'ailleurs pas forcément tous des mercenaires, même si le contexte du Sur la Paix nous oriente en ce sens) en proie à la

\footnotetext{
${ }^{101}$ Isocrate lui-même ne l'utilise que 2 fois, ici et dans le Plataïque, 55, où le locuteur se lamente sur le sort de celui qui est sans patrie. On trouve l'adjectif 14 fois chez les Tragiques (dont 10 occurrences chez Euripide). Il est au contraire d'un emploi rare chez les orateurs (Antiphon, Tétralogie 2, 9, [Lysias], Pour Polystratos, 35, Démosthène, C. Euboulidès, 70).

${ }^{102}$ Ces mots prennent aussi un sens tout à fait abstrait chez Isocrate, avec une connotation négative : ils désignent l'égarement de l'esprit ou l'errance intellectuelle (Evagoras, 43, Lettre VI Aux fils de Jason, 10, Aréopagitique, 77, Sur l'échange, 52, 265, Panathénaïque, 89, 95), voire un excursus dans un discours (C. les sophistes, 15).

${ }^{103} \mathrm{Il}$ arrive même que des troupes grecques combattent les unes contre les autres, comme précisément du-

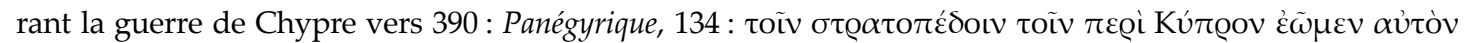

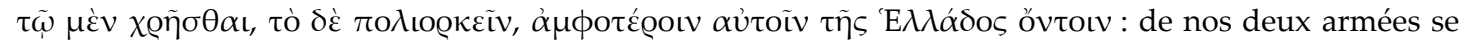
trouvant à Chypre, nous laissons le grand Roi en utiliser une et assiéger l'autre, quand toutes deux sont grecques. Cet épisode pose la question de l'unité ou non des troupes mercenaires en Grèce. Trundle 2004, p. 147, souligne la vision idéaliste d'Isocrate qui espère une union militaire des Grecs contre les barbares. Je me demande quant à moi si Isocrate était à ce point naïf ou idéaliste.

104 Voir supra pour les commentaires de ce passage.

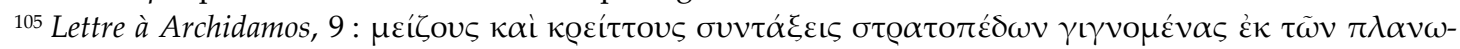

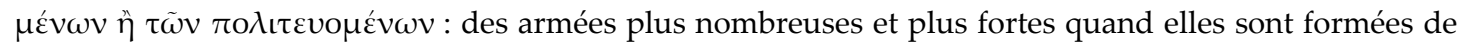
vagabonds plutôt que de citoyens.

${ }^{106}$ Ce sur quoi insiste largement Aymard 1967, p. 488 : «L'apparition et le développement du mercenariat permettent donc de diagnostiquer l'existence d'une sérieuse crise économique et sociale : il ne constitue qu'un pis-aller pour les plus valides des hommes que talonne la misère ». Fuks 1972 a été aussi l'un des premiers à souligner l'importance de la question socio-économique dans la pensée d'Isocrate. Il analyse le tableau, terrifiant, qu'Isocrate brosse de la situation de la Grèce dans les années 360, ainsi que les rap-

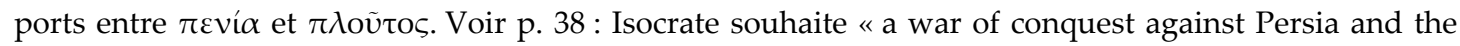
settlement of those unable to find livehood in Greece, the ejected and the displaced in and out the poleis of Greece, in the conquered territory ». Voir aussi Soesbergen 1982-1983, p. 133, qui insiste sur le lien existant entre la pauvreté et le mercenariat.
} 


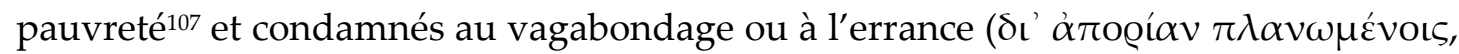
24) : cette fois, le vagabondage n'est pas (seulement) perçu comme un fléau pour les autres. L'errance, le déracinement, sont certes le fait d'hommes sans patrie, criminels et cruels, auxquels serait resté étranger Timothée ${ }^{108}$, mais ces malheurs sont expliqués par des considérations tout autres, non pas exclusivement morales. Cette approche sera d'ailleurs reprise dans le Philippe (voir infra). Je noterai pour l'instant, et après L. Marinovič ${ }^{109}$, que dans ce discours le ton a changé et, contrairement à ce que nous venons de lire dans la lettre à Archidamos, les vagabonds représentent alors un vivier commode, dans lequel Philippe pourrait puiser facilement pour se constituer une ar-

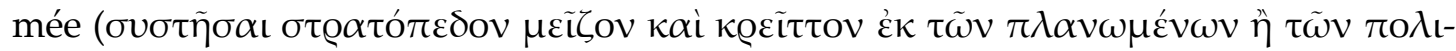
$\tau \varepsilon v o \mu \varepsilon ́ v \omega v, 96)$. Un peu plus loin, Isocrate évoque ces vagabonds et déclare que ceuxci doivent leur errance à un manque quotidien de moyens pour vivre : toù

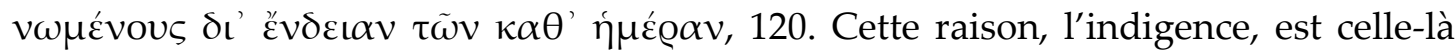

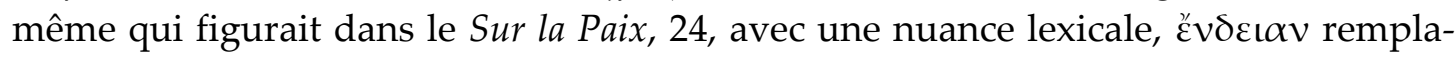
çant ici ảrooí $\alpha$ v.

La question est de savoir précisément ce qui poussait ces hommes à errer. Etait-ce la seule indigence ? Pouvons-nous affirmer que l'analyse - toute succincte est-elle que fait Isocrate en 355 et 346 est la même que celle que nous trouvions une trentaine d'années plus tôt, en 380, dans le Panégyrique, 146, où le substantif employé était $\phi \alpha v$ -

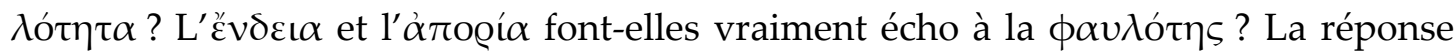

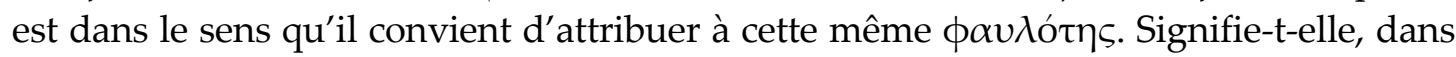
le Panégyrique, une condition modeste, économiquement difficile? Si oui, les textes de 355 et de 346 poursuivent l'idée exprimée en 380, en incriminant une situation socio-économique défavorable. Isocrate explique dans ce cas le phénomène du mercenariat, dès le Panégyrique, en dédouanant - au moins partiellement - les hommes

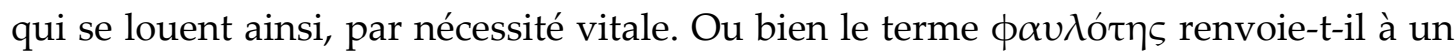
mauvais état d'esprit ? Si oui, Isocrate attaque ces mêmes individus sur le plan moral. Ludmila Marinovič a tenté de répondre, sans vraiment trancher, en considérant que selon les traductions, «l'accent est [...] mis soit sur l'aspect moral, soit sur l'aspect économique du terme ». Pour L. Marinovič, le sens économique ne va pas de soi, car Isocrate utilise d'autres termes pour désigner les gens dans le besoin [voir Sur la Paix, $24: \dot{\alpha} \pi$ oqí $\alpha v]$. «Tout ce qui vient d'être dit nous amène à penser qu'Isocrate n'avait pas en vue la pauvreté, ou en tout cas pas seulement la pauvreté : bien plutôt une difficulté de situation, au sens large du terme $»^{110}$. Je pencherais personnellement

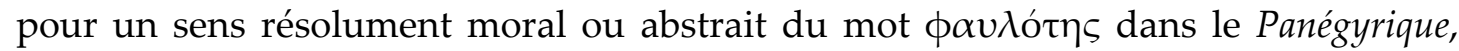
146. Trois arguments m'y poussent. D'abord, - et en accord avec L. Marinovič quand Isocrate veut indiquer la pauvreté matérielle, il est sans ambiguïté, comme

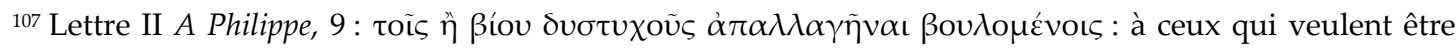
débarrassés d'une vie de malheurs. Nous avons un discours à peu près semblable chez Démosthène, Sur les Symmories, 31 : les mercenaires sont des hommes qui se louent pour échapper à leur pauvreté : $\dot{\alpha} \pi \alpha \lambda$ -

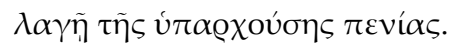

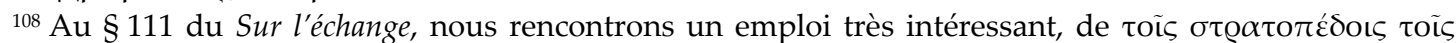
$\pi \lambda \alpha \nu \omega \mu \varepsilon ́ v o ı \varsigma$ : Timothée n'est pas rompu au métier de ces armées errantes.

${ }_{109}$ Marinovič 1988, p. 261, note bien le changement de perspective chez Isocrate entre le Panégyrique et le Philippe. Dans le Philippe (90-92), «il dépeint ses mercenaires grecs (ceux de Cyrus) sous d'autres couleurs que dans le Panégyrique - simplement comme de vaillants soldats. »

${ }^{110}$ Marinovič 1988, pp. 243-244.
} 


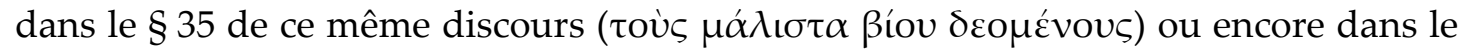

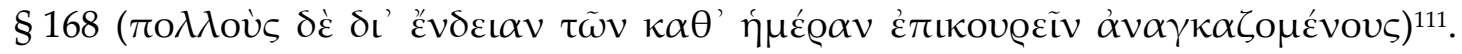
Ensuite, je pense que l'on doit privilégier le sens moral et abstrait de $\phi \alpha u \lambda o ́ \tau \eta \varsigma$, car il est bien présent chez les contemporains d'Isocrate. Pour ne prendre que quelques exemples, Xénophon utilise le terme pour désigner l'ignorance d'Euthydème à propos de la question du tyran ${ }^{112}$, Démosthène parle de l'impéritie éventuelle des stratèges $^{113}$, Aristote considère que la tyrannie est une perversion de la monarchie ${ }^{114}$. Enfin, le texte même du Panégyrique, en jouant sur une antithèse (adverbe ḋ@ı $\delta ı \grave{\alpha} \phi \alpha v \lambda o ́ \tau \eta \tau ')$ indique clairement que les mercenaires ne sont pas choisis pour leur valeur intrinsèque ou pour leurs qualités, mais que ce sont des défaillances personnelles et/ou un mauvais état d'esprit ${ }^{115}$ qui les conduisent à se louer. Si cette analyse lexicale est fondée, cela signifie qu'Isocrate a évolué dans son appréciation des mer-

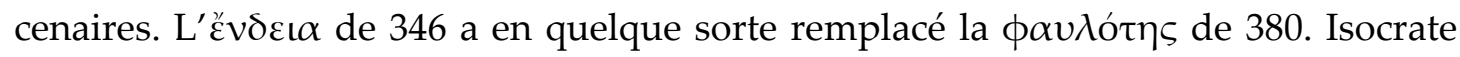
prend davantage en compte les difficultés matérielles de ces hommes, qu'il avait autrefois jugés comme des incapables dans leur vie sociale. Bien sûr, l'indigence peut toujours apparaître comme la conséquence de mauvaises prédispositions, comme on le lit encore en 355 dans le Sur la Paix, 79 - où les mercenaires sont définis comme des paresseux et des délinquants ${ }^{116}$-, mais on trouve surtout ce genre d'argument, à savoir l'affirmation d'un lien de cause à effet entre la pauvreté et les mauvaises manières, quand il y a volonté de nuire ou d'abattre un adversaire devant un jury : ainsi faisait Démosthène quand il dénigrait Eschine et se moquait de son enfance « pauvre »117. Il n'est pas certain qu'une telle intention de condamner les mercenaires à travers leur pauvreté soit présente chez Isocrate en 346 . Il cherche plutôt une solution à leur misère en reprenant son programme de colonisation, moyen de fixer outre-mer ces hommes, même si cela doit servir avant tout les intérêts des Grecs et ceux de Philippe lui-même.

Le vagabondage des mercenaires pose enfin la question de leur solitude ${ }^{118}$ ou non. Sont-ils accompagnés de leurs proches, ou bien sont-ils en rupture, non seulement avec leur cité, mais encore avec leurs familles? Les commentateurs n'ont sans doute pas assez insisté sur cet aspect chez Isocrate ${ }^{119}$, trop vite jugé comme un accusateur de

\footnotetext{
${ }^{111}$ Beaucoup étant contraints de servir [comme mercenaires] parce qu'ils n'ont pas de quoi vivre au quotidien.

112 Xénophon, Mémorables, IV, 2, 39.

113 Démosthène, Sur la Couronne, 303.

${ }^{114}$ Aristote, Ethique à Nicomaque, $1160 \mathrm{~b}$.

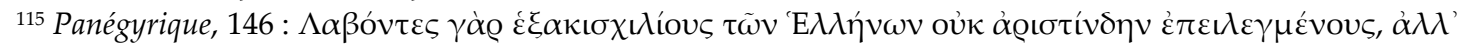

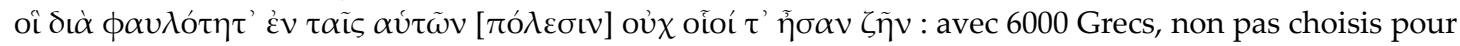
leur excellence, mais des hommes que leurs défauts empêchaient de vivre dans leur propre cité. Voir

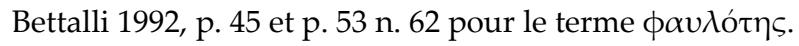

${ }^{116}$ Voir supra, note 68.

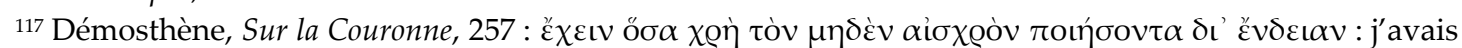
tout ce qu'il faut à quelqu'un pour ne pas être poussé au mal à cause de l'indigence.

${ }^{118}$ Dans un domaine un peu différent, Xénophon insiste sur la solitude qui menace les mercenaires conduits par Cléarque. Celui-ci dit en effet à Tissapherne que des troupes abandonnées à elles-mêmes, sans

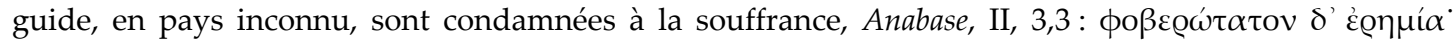

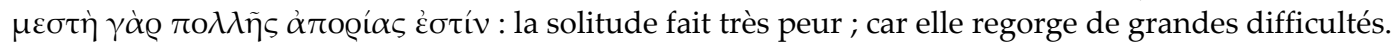

${ }^{119}$ Marinovič 1988 accorde quelques lignes à cette question, pp. 266-267 : p. 266 : «Isocrate les (= mercenaires) considère subjectivement : de façon négative, avec mépris et en même temps un certain effroi, mais
} 
mercenaires méprisés ou redoutés. Il lui arrive au contraire de traiter de la solitude du mercenaire ou de sa famille, de ces déracinés. Il est d'ailleurs révélateur que jamais Isocrate ne fasse état d'une quelconque solidarité entre les mercenaires euxmêmes, comme cela a pourtant dû exister ${ }^{120}$. Pour lui, les armées de mercenaires seraient composées de solitudes rassemblées, de vagabonds à la dérive, comme l'a montré le vocabulaire étudié plus haut. La mention des femmes et des enfants est certainement à double tranchant: elle peut soit, avec tout le pathos de la rhétorique, insister sur les crimes commis par ces hommes, soit au contraire attirer sur ces mêmes hommes une forme de sympathie, de compassion. Il faut voir en effet la manière dont Isocrate s'apitoie sur le sort des femmes et des enfants pour dénoncer la cruauté des mercenaires : dans 1'A Archidamos, il brosse un tableau réaliste et pathétique, et relativement inaccoutumé chez lui, des cruautés subies par ces populations sans défense ${ }^{121}$, des populations qui devraient pourtant être chéries, protégées et respectées plus que tout au monde, comme nous le lisons dans le Nicoclès ${ }^{122}$.

Cela n'empêche qu'Isocrate s'apitoie aussi sur les familles des mercenaires, et, à travers elles, sur les mercenaires eux-mêmes. D'abord, dans l'Eginétique, en 391-390, il s'indigne de voir une sœur ne pas pleurer son frère mort à l'étranger (sans doute comme mercenaire) ${ }^{123}$. Nous avons là une espèce de contre-exemple, d'événement contre-nature, destiné à provoquer la réprobation et la mise en accusation de cette femme. Ensuite, Isocrate écrit dans le Panégyrique que des familles entières ${ }^{124}$, ruinées à la suite de guerres intestines ou non, sont condamnées à errer, et que des hommes doivent alors se louer comme mercenaires. Ce même passage du Panégyrique va encore plus loin, puisque Isocrate s'indigne de voir les Grecs ne pas prendre en pitié ces malheureux ${ }^{125}$. Le traitement rhétorique de cette colère - sincère ou non, il est difficile d'en juger - est très appuyé : Isocrate oppose la littérature qui émeut, en faisant allusion à des vers de Tyrtée ${ }^{126}$, à la réalité, qui malgré sa dureté, laisse indifférent.

aussi avec compréhension, en pensant à leur destinée pitoyable et peu enviable ». Voir aussi Trundle 2004, pp. 141-142.

120 Sartre 2006, p. 288 : «Le politeuma se substitue à la famille absente ou inexistante, le cas échéant ", à propos des mercenaires de Sidon à la fin du IIIe-début du IIe siècles.

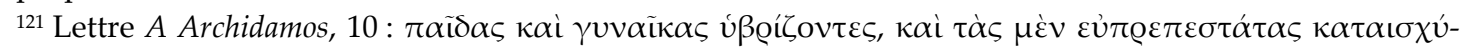
vov $\tau \varepsilon \varsigma$, outrageant enfants et femmes, déshonorant les plus distinguées d'entre elles.

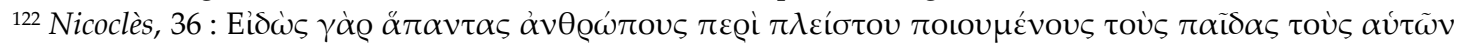

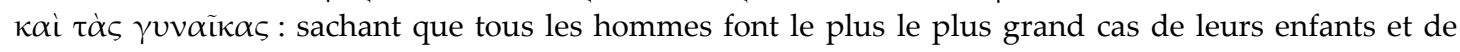

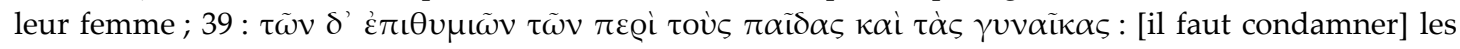

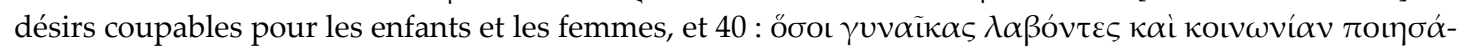

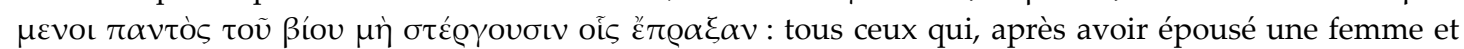
l'avoir associée à tous les instants de leur vie, ne trouvent aucun plaisir à ce qu'elle fait.

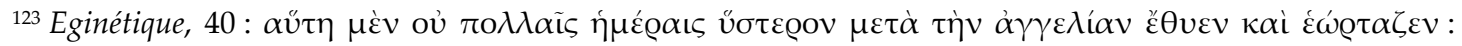
peu de jours après cette nouvelle [la mort du frère en Lycie], elle sacrifiait et célébrait une fête ; et ỏ $\lambda i ́ \gamma o v$

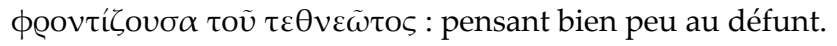

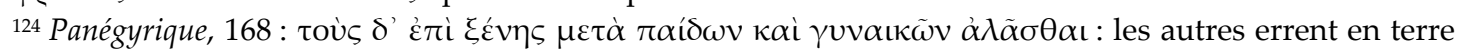
étrangère avec enfants et femmes. Voir supra, note 61.

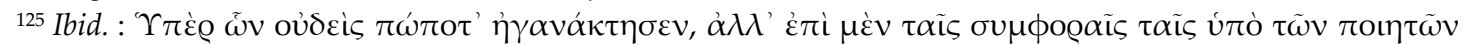

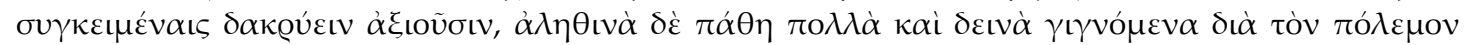

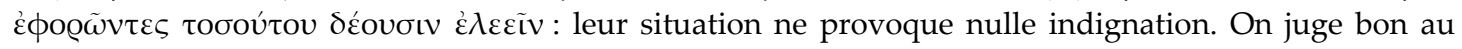
contraire de pleurer sur les malheurs représentés par les poètes, tandis que le spectacle des vraies souffrances, nombreuses et terribles, est si loin d'apitoyer que...

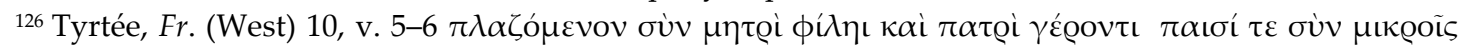

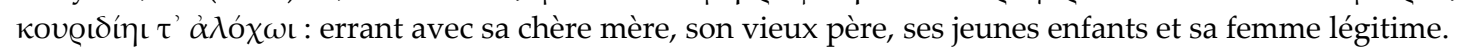


Toujours dans le Panégyrique, dans la partie nettement tournée contre Sparte, Isocrate dénonce le traitement infligé à ces Grecs d'Asie mineure, obligés, à la suite du traité d'Antalcidas, de servir dans les armées du Roi contre d'autres Grecs. Aucun terme ne désigne ces hommes comme des mercenaires, mais c'est implicite ${ }^{127}$. Un peu plus tard, sans qu'il soit question des seuls mercenaires, dans le Plataïque, le locuteur exprime devant les Athéniens le drame que représentent à la fois la séparation familiale et l'exil dû à la pauvreté128.

Il apparaît ainsi que le vagabondage est un thème qui peut inquiéter, mais qui, également, provoque une certaine compassion. On peut toujours se demander si ce sentiment est sincère ou non, s'il n'est pas mis en avant en fonction du destinataire du discours ou de la lettre, et selon les circonstances. Il n'en reste pas moins qu'il est sollicité, et de façon assez remarquable.

Il ressort de cette étude lexicale du corpus isocratique d'abord que la question du mercenariat ne saurait être réduite à un jugement négatif. Les mercenaires sont des hommes sans doute cruels, certainement dangereux pour les cités et les populations grecques, mais ce sont aussi de bons soldats (en cela Isocrate est assez proche de Xénophon), et, également des victimes des difficultés socio-économiques de ces mêmes cités. Et avec eux, ce sont leurs familles qui souffrent.

Ensuite, les techniques de guerre ont évolué au cours du IVe siècle, et avec elles, le regard d'un Isocrate, qui, en 380 et surtout en 355, dénonçait les exactions commises par ces troupes et la démission de citoyens fuyant leurs obligations militaires, et qui, en 346, recommande à Philippe de recourir, sans difficulté qui plus est, à ces mercenaires.

Il semble enfin que le critère politique aide à expliquer la position d'Isocrate en la matière. Pour bien apprécier son jugement, il me semble nécessaire de distinguer les démocraties et les régimes personnels. Cette distinction est d'ailleurs permanente dans la majeure partie des textes d'Isocrate. Si le recours à des troupes aguerries et formées, donc à des mercenaires, est souvent et plutôt condamnable dans une démocratie comme Athènes, il est tout à fait admis dans des royautés (perse ou macédonienne). La preuve : si Athènes emploie des mercenaires, elle se comporte comme le grand Roi, donc comme le roi des barbares (Sur la Paix). Il n'empêche que cette vue

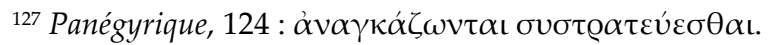

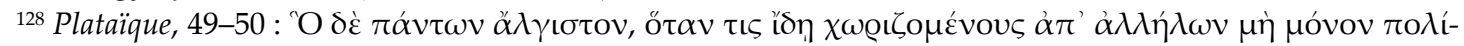

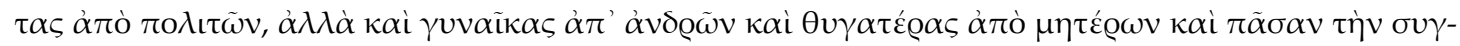

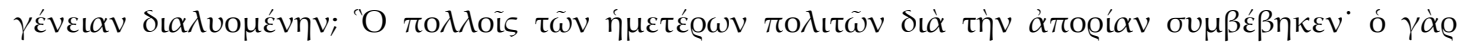

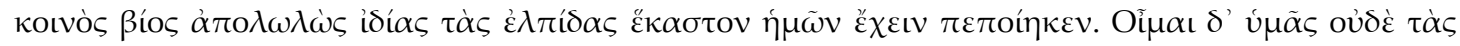

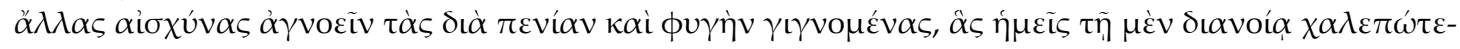

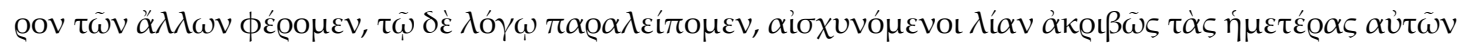

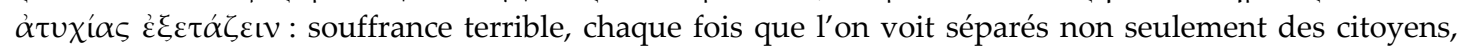
mais aussi des femmes éloignées de leurs maris, des filles de leurs mères, toute la famille dispersée ? Ce qui est arrivé à nombre de nos concitoyens, à cause de la pauvreté ; la fin de la vie familiale a conduit chacun de nous à nourrir des espérances égoïstes. Et je pense que vous n'ignorez pas non plus les autres hontes qui naissent de la misère et de l'exil ; si, dans notre cœur, nous les supportons plus difficilement que les autres, au contraire nous n'en parlons pas, trop honteux d'exposer par le détail nos propres infortunes.
} 
doit être nuancée : le roi de Macédoine, lui, aurait toute légitimité pour recruter ce genre de troupes. Il y est même encouragé dans le Philippe. Il est vrai qu'avec la paix de Philocrate les Athéniens - Isocrate l'un des premiers - savent à qui ils ont affaire en la personne de Philippe. Cela signifie tout à la fois qu'Isocrate a évolué dans son jugement, qu'il a compris que l'on ne peut plus vraiment se passer de ces troupes, surtout quand elles sont grecques, destinées à combattre contre les barbares - et que le clivage politique - démocratie/royauté - ne fonctionne plus à partir de 346.

La question politique est enfin en lien avec celle de la nécessité ou non d'une professionnalisation des troupes. Autrement dit, peut-on établir un rapport entre le jugement que porte Isocrate sur le mercenariat, sur des troupes professionnelles, et son attachement ou non à la démocratie ? Le discours d'Isocrate est trop fluctuant, trop attaché aux circonstances et à son destinataire du moment pour que nous puissions répondre de façon certaine et systématique. Il est cependant possible d'admettre que, lorsqu'il condamne le recours à des troupes mercenaires, Isocrate ne se montre pas du tout anti-démocrate ; au contraire, puisqu'il reste attaché à l'idée du citoyen-soldat. Encore une fois, les rois peuvent recruter ce genre de personnel militaire, alors qu'une telle pratique risque souvent d'être perçue comme une déviance du fonctionnement démocratique, voire en contradiction avec lui, du moins selon Isocrate.

Christian Bouchet

Université des Antilles-Guyane

UMR 7041 Paris-X Nanterre

E-mail: christian.bouchet07@gmail.com 


\section{Vocabulaire et fréquence du thème du mercenaire ${ }^{129}$}

\begin{tabular}{|c|c|c|c|c|c|}
\hline & Soldat & Stipendié & Etranger & Vagabond & $\begin{array}{c}\text { Allusions, } \\
\text { périphrases... }\end{array}$ \\
\hline $\begin{array}{l}\text { C. Callimakhos } \\
(402-401)\end{array}$ & & 60 & & & \\
\hline $\begin{array}{l}\text { Eginétique (391- } \\
390)\end{array}$ & 38 & & & & \\
\hline $\begin{array}{l}\text { Eloge d'Hélène } \\
(390-380)\end{array}$ & & & 37 & & \\
\hline Panégyrique (380) & $\begin{array}{l}115.124 . \\
134 . \\
141.144 . \\
145 . \\
147 . \\
149 . \\
168 . \\
\end{array}$ & 142.153. & & $168 ?$ & $\begin{array}{l}\text { 35. } 124.146 . \\
185 \text { (plutôt } \\
\text { alliés?). }\end{array}$ \\
\hline Plataïque (371) & & & & & $\begin{array}{l}49-50 \text { : ceux } \\
\text { qui nourris- } \\
\text { sent des espé- } \\
\text { rances égoïs- } \\
\text { tes. }\end{array}$ \\
\hline Evagoras (365-362) & & & & 28 & \\
\hline Archidamos (366) & & & 76 & & \\
\hline $\begin{array}{l}\text { Lettre à Archidamos } \\
\text { (356) }\end{array}$ & & & & 9. & \\
\hline $\begin{array}{l}\text { Sur la Paix (356- } \\
355)\end{array}$ & 118. & $\begin{array}{l}44.47 . \\
112 .\end{array}$ & 24.46 .48$. & 24. & $\begin{array}{l}46 \text { : les enne- } \\
\text { mis communs } \\
\text { à tous les } \\
\text { hommes. } \\
79: \text { les plus } \\
\text { paresseux. }\end{array}$ \\
\hline $\begin{array}{l}\text { Aréopagitique (354 } \\
\text { ou 357/356) }\end{array}$ & & & 9. & & $\begin{array}{l}\text { 54. Ceux des } \\
\text { Grecs que les } \\
\text { Athéniens } \\
\text { paient pour } \\
\text { servir dans la } \\
\text { flotte (ra- } \\
\text { meurs). }\end{array}$ \\
\hline Sur l'échange (353) & 111. & 111.120. & 116. & 111. & \\
\hline Philippe (346) & 125.126. & 55.91. & $\begin{array}{l}\text { 96. 96bis. } \\
122 .\end{array}$ & 96. 120. & 126. \\
\hline $\begin{array}{l}\text { Lettre II A Philippe } \\
(344)\end{array}$ & & 9.19. & 19. & & \\
\hline $\begin{array}{l}\text { Panathénaïque } \\
\text { (342-339) }\end{array}$ & 81 & $82.116 ?$ & 186. & & 79 \\
\hline
\end{tabular}

${ }^{129}$ Ce tableau fait apparaître une certaine évolution dans la présentation du mercenaire. Le traitement change à partir des années 350. Le mercenaire est un soldat surtout jusque vers 353. Ensuite, il est plutôt présenté comme un stipendié étranger et vagabond. 


\section{BIBLIOGRAPHIE}

Aymard, A. (1967) «Mercenariat et histoire grecque. » - Etudes d'histoire ancienne. Paris : PUF, 487-498.

Azoulay, V. (2006) « L'Archidamos d'Isocrate : une politique de l'espace et du temps ». - Revue des études grecques 119, 504-531.

Bearzot, C. (2001) « Xenoi e profughi nell’Europa di Isocrate». - Urso, G. (éd.), Integrazione, mescolanza, rifiuto : incontri di popoli, lingue e culture in Europa dall'antichità all'umanesimo. Atti del convegno internazionale, Cividale del Friuli, 21-23 settembre 2000. Roma : 'L'Erma' di Bretschneider, 47-63.

Bettalli, M. (1992) «Isocrate e la guerra ». - Opus 11, 37-56.

Bettalli, M. (2006a) «L'immagine del mercenario nella Grecia del IV secolo a.C. ». Vaggioli, Maria Adelaide (éd.), Guerra e pace in Sicilia e nel Mediterraneo antico (VIIIIII sec. a.C.). Arte, prassi e teoria della pace e della guerra, vol. I. Pisa : Edizioni della Normale, 19-28.

Bettalli, M. (2006b) « Hoi ton Hellenon aporoi : I mercenari del mondo greco classico tra violenza, emarginazione e integrazione ». - Urso, G. (éd.), Terror et Pavor : violen$z a$, intimidazione, clandestinità nel mondo antico. Atti del convegno internazionale, Cividale del Friuli, 22-24 settembre 2005. Pisa : ETS, 55-64.

Bianco, E. (2003) «De Isocratis malignitate». - Orth, W. (éd.), Isokrates. Neue Ansätze zur Bewertung eines politischen Schriftstellers des 4. Jhdts. V. Chr. Trier : WVT, 128-215.

Bianco, E. (2007) Lo stratego Timoteo torre di Atene. Alessandria : Edizioni dell'Orso.

Blass, F. ; Benseler, G. (1885-1889) Isocratis Orationes. Leipzig: Teubner.

Bouchet, C. (2007) «Conon, navarque perse à Cnide, 394 ? ». - Rivista di cultura classica e medioevale 49.2, 231-247.

Burckhardt, L. A. (1996) Bürger und Soldaten: Aspekte der politischen und militarischen Rolle athenischer Bürger im Kriegswesen des 4. Jahrhunderts v. Ch. (Historia Einzelschriften; 101.) Stuttgart : Steiner.

Cawkwell, G. L. (1981) «The failure of the Second Athenian Confederacy ». - Journal of Hellenic Studies 101, 40-55.

Ceccarelli, P. (1993) «Sans thalassocratie, pas de démocratie? Le rapport entre thalassocratie et démocratie à Athènes dans la discussion du Ve et IVe siècle av. J.-C ». - Historia 42, 444-470.

Chankowski, A. S. (1997) «Compte rendu de Bürger und Soldaten de L. A. Burckhardt». - Topoi 7, 331-348.

Chantraine, P. (1968) Dictionnaire étymologique de la langue grecque, II. Paris : Klincksieck.

Cloché, P. (1963) Isocrate et son temps. Besançon: Annales littéraires de l'université de Besançon.

Ducrey, P. (2000) « Les aspects économiques de l'usage des mercenaires dans la guerre en Grèce ancienne : avantages et inconvénients du recours à une main-d'œuvre militaire rémunérée ». - Entretiens d'archéologie et d'histoire. Economie antique. La guerre dans les économies antiques. Saint-Bertrand-de-Comminges: Musée archéologique départemental, 197-207.

Froidefond, C. (1971) Le mirage égyptien dans la littérature grecque d'Homère à Aristote. Paris : Publications de la Sorbonne.

Fuks, A. (1972) «Isokrates and the social-economic situation in Greece ». - Ancient Society 3, 17-44. 
Garlan, Y. (1999) Guerre et économie en Grèce ancienne. Paris : La Découverte.

Harding, P. (1995) « Athenian foreign policy in the fourth century ». - Klio 77,105-125.

Hunt, P. (2007) « Military forces ». - Sabin, P. ; Van Wees, H. ; Whitby, M. (éd.), The Cambridge history of Greek and Roman warfare, I. Cambridge : Cambridge University Press,108-146.

Krasilnikoff, J. A. (1992) «Aegean mercenaries in the fourth to second centuries B.C.: a study in payment, plunder and logistics of ancient Greek armies ». - Classica et Mediaevalia 43, 23-36.

Krasilnikoff, J. A. (1993) «The regular payment of Aegean mercenaries in the classical period ». - Classica et Mediaevalia 44, 77-95.

Lengauer, W. (1979) Greek commanders in the 5th and 4th century B.C. Politics and ideology : a study of militarism. Warszawa : wyd. uniwersytetu Warszawskiego.

Mandilaras, B. G. (éd.) (2003) Isocrates. Opera omnia, 3 vol. Leipzig : Saur.

Marinovič, L. (1998) Le mercenariat grec au IVe siècle avant notre ère et la crise de la polis. Paris : Les Belles Lettres.

Mathieu, G. (1925) Les idées politiques d'Isocrate. Paris : Les Belles Lettres.

Mathieu, G. ; Brémond, E. (éd.) (1928-1962) Isocrate, 4 vol. Paris : Les Belles Lettres.

Mossé, C. (1953) «Sur un passage de 1' Archidamos d'Isocrate ». - Revue des études anciennes 55, 29-35.

Mossé, C. (1976) «Les salariés à Athènes au IVe siècle ». - Dialogues d'histoire ancienne $2,97-101$.

Nouhaud, M. (1982) L'utilisation de l'histoire par les orateurs attiques. Paris: Les Belles Lettres.

Papillon, T. L. (2004) Isocrates II. Austin : University of Texas Press.

Parke, H. W. (1933, éd. de 1970) Greek mercenary soldiers : from the earliest times to the Battle of Ipsus. Oxford : Clarendon Press.

Pritchett, W. K. (1991) The Greek state at war, V. Berkeley : University of California Press.

Pritchett, W. K. (1992) The Greek state at war, II. Berkeley : University of California Press.

Riedinger, J.-C. (1991) Etude sur les Helléniques : Xénophon et l'histoire. Paris : Les Belles Lettres.

Romilly, J. de (1954) «Les modérés athéniens vers le milieu du IVe siècle ». - Revue des études grecques 67, 327-354.

Sartre, M. (2006) «Stèles de mercenaires de Sidon, ou L'armée et la guerre dans le monde hellénistique ». - Histoires grecques. Paris : Seuil, 283-291.

Simonetti Agostinetti, A. (2002) «Coloni greci nell' Asia orientale : problemi di identità negli insediamenti coloniali creati da Alessandro Magno ». - Moscati Castelnuovo, L. (éd.), Identità e Prassi Storica nel Mediterraneo Greco. Milano : Edizioni ET, 205-216.

Soesbergen, P. G. van (1982-1983) «Colonisation as a solution to social-economic problems in fourth-century Greece. A confrontation of Isocrates with Xenophon ». - Ancient Society 13-14, 131-145.

Trédé, M. (1992) Kairos. L'à-propos et l'occasion. Paris : Klincksieck.

Trundle, M. (2004) Greek mercenaries. London \& New-York : Routledge.

Wees, H. van (2004) Greek warfare: myths and realities. London : Duckworth.

Will, E. (1975) «Notes sur $\mathrm{MI} \Sigma \Theta \mathrm{O} \Sigma »$. - Hommages à Claire Préaux. Bruxelles: éditions de l'Univ. de Bruxelles, 426-438.

Yalichev, S. (1997) Mercenaries of the ancient world. London : Constable. 